\title{
Unraveling the Growth Mechanism of Magic-Sized Semiconductor Nanocrystals
}

\author{
Aniket S. Mule,${ }^{\S}$, Sergio Mazzotti $,{ }^{\S}, \dagger$ Aurelio A. Rossinelli,${ }^{\dagger}$ Marianne Aellen,${ }^{\dagger}$ P. Tim Prins,${ }^{\ddagger}$ \\ Johanna C. van der Bok, ${ }^{\ddagger}$ Simon F. Solari, ${ }^{\dagger}$ Yannik M. Glauser, ${ }^{\dagger}$ Priyank V. Kumar ${ }^{\dagger, \nabla}$ \\ Andreas Riedinger, ${ }^{\dagger}, \|$ and David J. Norris ${ }^{*}, \dagger$
}

${ }^{\dagger}$ Optical Materials Engineering Laboratory, Department of Mechanical and Process Engineering, ETH Zurich, 8092 Zurich, Switzerland
${ }^{\ddagger}$ Condensed Matter and Interfaces, Debye Institute for Nanomaterials Science, Utrecht University, 3508 TA Utrecht, The Netherlands

DPresent Address: School of Chemical Engineering, University of New South Wales, Sydney 2052, Australia

"Max-Planck-Institute for Polymer Research, Ackermannweg 10, 55128 Mainz, Germany

\section{S1 EXPERIMENTAL DETAILS}

\section{S1.1 Synthetic methods of magic-sized nanocrystals (MSNCs) Materials}

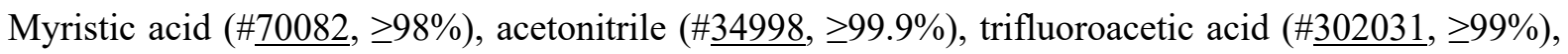
trifluoroacetic anhydride (\#106232, $\geq 99 \%$ ), selenium (Se, \#209651, $\geq 99.5 \%$ ), lithium aluminum

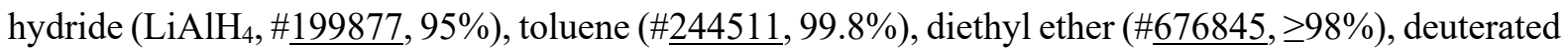
chloroform ( $\mathrm{CDCl}_{3}, \# \underline{236918}, 99.6$ atom \% deuterium), 1-octadecene (ODE, \#0806, 90\%), oleic acid (\#364525, 90\%), cadmium acetate dihydrate (\#289159, 98\%), cadmium chloride (technical grade, \#655198), and methyl acetate ( $\geq 98 \%$, \#W267600) were purchased from Sigma Aldrich. Triethyl amine (\#157910010, 99\%) and tetrahydrofuran (THF, \#348450010, 99.5\%) were purchased from Acros Organics. Cadmium oxide (\#48-0800, 99.999\%-Cd) was purchased from Strem Chemicals. Stearoyl chloride (\#S0404, >97\%) was purchased from TCI chemicals. Sodium chloride ( $\mathrm{NaCl}, \# 85139.360)$ was purchased from VWR chemicals. Hexane, methanol, and 2-propanol were purchased from Thommen-Furler AG. Absolute ethanol was purchased from Alcosuisse AG. All chemicals were used as received. Deionized (DI) water was obtained from a Milli-Q Advantage A10 water purification system (Merck Millipore). 


\section{Synthesis of cadmium myristate}

Cadmium myristate was synthesized by modifying the synthesis of lead oleate carried out by Hendricks et al. ${ }^{\mathrm{S} 1}$ Briefly, $44.8 \mathrm{mmol}$ (5.75 g) of cadmium (II) oxide was mixed with $20 \mathrm{~mL}$ of acetonitrile in a $100-\mathrm{mL}$ three-neck round-bottomed flask. The mixture was stirred at $0^{\circ} \mathrm{C}$ for $10 \mathrm{~min} .8 .96 \mathrm{mmol}$ $(0.7 \mathrm{~mL}, 0.2$ equiv.) of trifluoroacetic acid and $44.8 \mathrm{mmol}(6.2 \mathrm{~mL}, 1$ equiv.) of trifluoroacetic anhydride are then added. After $15 \mathrm{~min}$, the brownish mixture is heated to $50{ }^{\circ} \mathrm{C}$ to obtain a clear cadmium trifluoroacetate solution. In a separate 500-mL Erlenmeyer flask, $90.05 \mathrm{mmol}$ of myristic acid (20.56 g, 2.01 equiv.), $101.25 \mathrm{mmol}$ of triethyl amine (10.246 g, 2.26 equiv.) were added to $180 \mathrm{~mL}$ of 2-propanol. The cadmium trifluoroacetate solution was then gradually added to the myristic acid solution with stirring. The resulting white precipitate of cadmium myristate was isolated by suction filtration using a glass fritted funnel. The filtrate was thoroughly washed further 3 times with methanol $(300 \mathrm{~mL})$ and then dried under vacuum at room temperature for $>12 \mathrm{~h}$. The final product was grinded into fine powder and stored under ambient conditions.

\section{Synthesis of bis(stearoyl) selenide}

Synthesis of bis(stearoyl) selenide was carried out by adapting a protocol reported by Koketsu et al. ${ }^{\text {S2 }}$ Briefly, a suspension of elemental Se $(1.92 \mathrm{~g}, 24 \mathrm{mmol})$ and dry THF $(200 \mathrm{~mL})$ was prepared in a 500-mL four-neck flask and kept at $-10{ }^{\circ} \mathrm{C}$ (ice bath with $\mathrm{NaCl}$ ) under $\mathrm{N}_{2} . \mathrm{LiAlH}_{4}(0.76 \mathrm{~g}, 20 \mathrm{mmol})$ was then added and the mixture was stirred to form LiAlHSeH. After $30 \mathrm{~min}, 20 \mathrm{mmol}(6.8 \mathrm{~mL})$ of stearoyl chloride was added over a span of $2 \mathrm{~min}$ to the solution of LiAlHSeH. This was repeated at 60 , 90 , and $120 \mathrm{~min}$. The reaction was stopped after $150 \mathrm{~min}$ by adding $5 \mathrm{~mL}$ of DI water. The ice bath was removed and the reaction mixture was diluted by $250 \mathrm{~mL}$ of diethyl ether. The reaction mixture was further washed 4 times with $150 \mathrm{~mL}$ of a saturated $\mathrm{NaCl}$ solution. The turbid mixture was heated to $\sim 50{ }^{\circ} \mathrm{C}$ to form a clear solution. The flask was left at room temperature for $>10 \mathrm{~h}$ to induce crystallization of the desired product. The recrystallized product was separated by filtration and vacuum dried for $>12 \mathrm{~h}$ at $25^{\circ} \mathrm{C}$. The product was stored in inert atmosphere $\left(\mathrm{N}_{2}\right)$ for further use. ${ }^{1} \mathrm{H}$ NMR $\left(\mathrm{CDCl}_{3}, 400 \mathrm{MHz}\right): \delta 0.88$ (t, 6H, $J=6.8 \mathrm{~Hz}$ ), 1.25 (s, 56H), 1.65 (quint, 4H, $J=7.5 \mathrm{~Hz}$ ), 2.81 (t, 4H, $J=7.4 \mathrm{~Hz}) .{ }^{13} \mathrm{C} \mathrm{NMR}\left(\mathrm{CDCl}_{3}, 400 \mathrm{MHz}\right): \delta 14.28,22.86,24.96,28.91,29.39,29.53,29.86,32.09$, 49.70, 198.34. ${ }^{77} \mathrm{Se} \mathrm{NMR}\left(\mathrm{CDCl}_{3}, 400 \mathrm{MHz}\right): \delta 809.94$.

\section{Synthesis of MSNC 434}

$0.3 \mathrm{mmol}(80 \mathrm{mg})$ of cadmium acetate dihydrate, $0.7 \mathrm{mmol}(197.7 \mathrm{mg}, 222 \mu \mathrm{L})$ of oleic acid, and $15 \mathrm{~mL}$ of ODE were mixed in a $50-\mathrm{mL}$ three-neck flask. The mixture was heated to $110^{\circ} \mathrm{C}$ and degassed under vacuum for $90 \mathrm{~min}$. After degassing, the mixture was heated to $180{ }^{\circ} \mathrm{C}$ under $\mathrm{N}_{2}$. Simultaneously, $0.15 \mathrm{mmol}\left(92 \mathrm{mg}\right.$ ) of bis(stearoyl) selenide was dissolved in $2 \mathrm{~mL}$ of toluene under $\mathrm{N}_{2}$. Mild heating $\left(\sim 35^{\circ} \mathrm{C}\right)$ may be employed to obtain complete dissolution. Once the cadmium oleate solution reached $180^{\circ} \mathrm{C}$, the solution of bis(stearoyl) selenide was rapidly injected into the hot mixture. The MSNCs 
were allowed to grow at $180^{\circ} \mathrm{C}$. After $25 \mathrm{~min}$, the mixture was rapidly cooled down to room temperature by placing the flask in a water bath. The reaction-mixture volume was increased to $\sim 30 \mathrm{~mL}$ by adding $15 \mathrm{~mL}$ of hexane and $1 \mathrm{~mL}$ of oleic acid. $150 \mathrm{~mL}$ of methyl acetate was added, and the sample was centrifuged at $8586 \mathrm{~g}(8000 \mathrm{rpm})$ for $5 \mathrm{~min}$. The precipitate was redispersed in $5 \mathrm{~mL}$ of hexane. The washing cycle was repeated once by adding $25 \mathrm{~mL}$ of methyl acetate, centrifuged, and the precipitate was dispersed in $5 \mathrm{~mL}$ of toluene. The final washing cycle was carried out by adding $2.5 \mathrm{~mL}$ of acetonitrile to the sample and carrying out the centrifugation at $8586 \mathrm{~g}(8000 \mathrm{rpm})$ for $5 \mathrm{~min}$. The final product (precipitate) was stored in $5 \mathrm{~mL}$ of hexane.

\section{Synthesis of MSNC 455}

The synthesis of MSNC 455 was very similar to MSNC 434 except for the synthesis temperature, the duration, and the steps associated with the subsequent cleaning. Briefly, $0.3 \mathrm{mmol}(80 \mathrm{mg})$ of cadmium acetate dihydrate, $0.7 \mathrm{mmol}(197.7 \mathrm{mg}, 222 \mu \mathrm{L})$ of oleic acid, and $15 \mathrm{~mL}$ of ODE were mixed in a 50$\mathrm{mL}$ three-neck flask. The mixture was heated to $110^{\circ} \mathrm{C}$ and degassed under vacuum for $90 \mathrm{~min}$. After degassing, the mixture was heated to $210^{\circ} \mathrm{C}$ under $\mathrm{N}_{2}$. Simultaneously, $0.15 \mathrm{mmol}(92 \mathrm{mg})$ of bis(stearoyl) selenide was dissolved in $2 \mathrm{~mL}$ of toluene under $\mathrm{N}_{2}$. Mild heating $\left(\sim 35^{\circ} \mathrm{C}\right)$ may be employed to obtain complete dissolution. Once the cadmium oleate solution reached $210^{\circ} \mathrm{C}$, the solution of bis(stearoyl) selenide was rapidly injected into the hot mixture. Note that the temperature drops to $190^{\circ} \mathrm{C}$ on injection of the selenium precursor. The system needs to be manually purged to return the temperature to $210{ }^{\circ} \mathrm{C}$ in $\sim 5 \mathrm{~min}$. This manual purge was done by taking a 1-mL syringe and pulling out $\sim 200 \mu \mathrm{L}$ of gas from the reaction flask every minute for a total of $5 \mathrm{~min}$. The reaction was stopped after $27 \mathrm{~min}$ and $30 \mathrm{~s}$ and the mixture was rapidly cooled to $\sim 180^{\circ} \mathrm{C}$ with an air gun and then with a water bath to room temperature. The reaction-mixture volume was increased to $\sim 30 \mathrm{~mL}$ by adding $15 \mathrm{~mL}$ of hexane and $1 \mathrm{~mL}$ of oleic acid. Since the reaction mixture in this case often contains a significant proportion of undesired smaller MSNC sizes, special care must be taken to ensure efficient separation in the first step of precipitation. $3 \mathrm{~mL}$ of ethanol was added to the reaction mixture. $\sim 100 \mathrm{~mL}$ of methyl acetate was then added in small quantities with continuous stirring until the solution turned turbid. As soon as this occurred, $6 \mathrm{~mL}$ of methyl acetate was added. The sample was centrifuged at $8586 \mathrm{~g}(8000 \mathrm{rpm})$ for $5 \mathrm{~min}$ and the precipitate containing the 455 species was redispersed in $5 \mathrm{~mL}$ of hexane. $1 \mathrm{~mL}$ of ethanol and $25 \mathrm{~mL}$ of methyl acetate was added, and the centrifugation was repeated at $8586 \mathrm{~g}(8000 \mathrm{rpm})$ for $5 \mathrm{~min}$. The precipitate was redispersed in $5 \mathrm{~mL}$ of toluene. The final washing cycle was carried out by adding $2.5 \mathrm{~mL}$ of acetonitrile to the sample and centrifuging at $8586 \mathrm{~g}$ (8000 rpm) for $5 \mathrm{~min}$. The final product (precipitate) was stored in $5 \mathrm{~mL}$ of hexane.

\section{Synthesis of MSNC 476}

The synthesis of MSNC 476 was very similar to MSNC 434 except for the synthesis temperature, the duration, and the steps associated with the subsequent cleaning. Briefly, $0.3 \mathrm{mmol}(80 \mathrm{mg})$ of cadmium 
acetate dihydrate, $0.7 \mathrm{mmol}(197.7 \mathrm{mg}, 222 \mu \mathrm{L})$ of oleic acid, and $15 \mathrm{~mL}$ of ODE were mixed in a 50 $\mathrm{mL}$ three-neck flask. The mixture was heated to $110^{\circ} \mathrm{C}$ and degassed under vacuum for $90 \mathrm{~min}$. After degassing, the mixture was heated to $210^{\circ} \mathrm{C}$ under $\mathrm{N}_{2}$. Simultaneously, $0.15 \mathrm{mmol}(92 \mathrm{mg})$ of bis(stearoyl) selenide was dissolved in $2 \mathrm{~mL}$ of toluene under $\mathrm{N}_{2}$. Mild heating $\left(\sim 35^{\circ} \mathrm{C}\right)$ may be employed to obtain complete dissolution. Once the cadmium oleate solution reached $210{ }^{\circ} \mathrm{C}$, the solution of bis(stearoyl) selenide was rapidly injected into the hot mixture. Note that the temperature drops to $190^{\circ} \mathrm{C}$ on injection of the selenium precursor. The system needs to be manually purged to return the temperature to $210{ }^{\circ} \mathrm{C}$ in $\sim 5 \mathrm{~min}$. This manual purge was done by taking a $1-\mathrm{mL}$ syringe and pulling out $\sim 200 \mu \mathrm{L}$ of gas from the reaction flask every minute for a total of $5 \mathrm{~min}$. The reaction was stopped after $2 \mathrm{~h}$ and the mixture was rapidly cooled to $\sim 180^{\circ} \mathrm{C}$ with an air gun and then with a water bath to room temperature. The reaction-mixture volume was increased to $\sim 30 \mathrm{~mL}$ by adding $15 \mathrm{~mL}$ of hexane and $1 \mathrm{~mL}$ of oleic acid. Subsequently, $5.5 \mathrm{~mL}$ of ethanol and $90 \mathrm{~mL}$ of methyl acetate were added to the reaction mixture. The sample was centrifuged at $8586 \mathrm{~g}(8000 \mathrm{rpm})$ for $5 \mathrm{~min}$, and the precipitate was collected and redispersed in $15 \mathrm{~mL}$ of hexane. $2.5 \mathrm{~mL}$ of ethanol and $45 \mathrm{~mL}$ of methyl acetate were added and centrifugation was repeated at $8586 \mathrm{~g}(8000 \mathrm{rpm})$ for $5 \mathrm{~min}$. The precipitate was redispersed in $6 \mathrm{~mL}$ of toluene, and $200 \mu \mathrm{L}$ of oleic acid was added. The final washing cycle was carried out by adding $3 \mathrm{~mL}$ of acetonitrile to the sample and centrifuging at $8586 \mathrm{~g}(8000 \mathrm{rpm})$ for $5 \mathrm{~min}$. The final product (precipitate) was stored in $5 \mathrm{~mL}$ of hexane.

\section{Synthesis of MSNC 494}

$0.6 \mathrm{mmol}(160 \mathrm{mg})$ of cadmium acetate dihydrate, $1.4 \mathrm{mmol}(395.4 \mathrm{mg}, 444 \mu \mathrm{L})$ of oleic acid, and $15 \mathrm{~mL}$ of ODE were mixed in a $50-\mathrm{mL}$ three-neck flask. The mixture was heated to $110^{\circ} \mathrm{C}$ and degassed under vacuum for $90 \mathrm{~min}$. After degassing, the mixture was heated to $240{ }^{\circ} \mathrm{C}$ under $\mathrm{N}_{2}$. Simultaneously, $0.15 \mathrm{mmol}\left(92 \mathrm{mg}\right.$ ) of bis(stearoyl) selenide was dissolved in $2 \mathrm{~mL}$ of toluene under $\mathrm{N}_{2}$. Mild heating $\left(\sim 35^{\circ} \mathrm{C}\right)$ may be employed to obtain complete dissolution. Once the cadmium oleate solution reached $240{ }^{\circ} \mathrm{C}$, the solution of bis(stearoyl) selenide was rapidly injected into the hot mixture. Note that the temperature drops to $200^{\circ} \mathrm{C}$ on injection of the selenium precursor. The system needs to be manually purged to return the temperature to $240{ }^{\circ} \mathrm{C}$ in $\sim 10 \mathrm{~min}$. This manual purge was done by taking a $1-\mathrm{mL}$ syringe and pulling out $\sim 200 \mu \mathrm{L}$ of gas from the reaction flask every minute for a total of $10 \mathrm{~min}$. The MSNCs were allowed to grow at $240{ }^{\circ} \mathrm{C}$. The reaction was stopped after $90 \mathrm{~min}$, and the mixture was rapidly cooled down to $\sim 180{ }^{\circ} \mathrm{C}$ with an air gun and then with a water bath to room temperature. The reaction-mixture volume was increased to $\sim 30 \mathrm{~mL}$ by adding $15 \mathrm{~mL}$ of hexane and $1 \mathrm{~mL}$ of oleic acid. Subsequently, $6 \mathrm{~mL}$ of ethanol and $75 \mathrm{~mL}$ of methyl acetate were added to the reaction mixture. The sample was centrifuged at $8586 \mathrm{~g}(8000 \mathrm{rpm})$ for $5 \mathrm{~min}$, and the precipitate was redispersed in $15 \mathrm{~mL}$ of hexane. $3 \mathrm{~mL}$ of ethanol and $30 \mathrm{~mL}$ of methyl acetate were added and centrifugation was repeated at $8586 \mathrm{~g}(8000 \mathrm{rpm})$ for $5 \mathrm{~min}$. The precipitate was redispersed in $6 \mathrm{~mL}$ of toluene and $200 \mu \mathrm{L}$ of oleic acid was added. The final washing cycle was carried out by adding $2.5 \mathrm{~mL}$ of acetonitrile to the sample 
and centrifuging it at $8586 \mathrm{~g}(8000 \mathrm{rpm})$ for $5 \mathrm{~min}$. The final product (precipitate) was stored in $5 \mathrm{~mL}$ of hexane.

Note: While the MSNC products so far have been synthesized using cadmium oleate (formed in situ) as the cadmium source, they can also be prepared using cadmium carboxylates. The exact parameters might vary slightly depending on which long-chain cadmium carboxylate is used. Small variations in outcomes are also observed with different batches of bis(stearoyl) selenide. These changes can be corrected for by slightly increasing or decreasing the duration of the synthesis.

\section{Synthesis of MSNC 554}

$1.31 \mathrm{mmol}(744.4 \mathrm{mg})$ of cadmium myristate, $0.38 \mathrm{mmol}(68.6 \mathrm{mg})$ of cadmium chloride, and $15 \mathrm{~mL}$ of ODE were mixed in a 50-mL three-neck flask. The mixture was heated to $110^{\circ} \mathrm{C}$ and degassed under vacuum for $30 \mathrm{~min}$. After degassing, the mixture was heated to $240{ }^{\circ} \mathrm{C}$ under $\mathrm{N}_{2}$. Simultaneously, $0.15 \mathrm{mmol}\left(92 \mathrm{mg}\right.$ ) of bis(stearoyl) selenide was dissolved in $2 \mathrm{~mL}$ of toluene under $\mathrm{N}_{2}$. Mild heating $\left(\sim 35^{\circ} \mathrm{C}\right)$ may be employed to obtain complete dissolution. Once the cadmium precursor reached $240{ }^{\circ} \mathrm{C}$, the solution of bis(stearoyl) selenide was rapidly injected into the hot mixture. Note that the temperature drops to $200{ }^{\circ} \mathrm{C}$ on injection of the selenium precursor. The system needs to be manually purged to return the temperature to $240{ }^{\circ} \mathrm{C}$ in $\sim 10 \mathrm{~min}$. This manual purge was done by taking a $1-\mathrm{mL}$ syringe and pulling out $\sim 200 \mu \mathrm{L}$ of gas from the reaction flask every minute for a total of $10 \mathrm{~min}$. The MSNCs were allowed to grow at $240{ }^{\circ} \mathrm{C}$. The reaction was stopped after $4 \mathrm{~h}$, and the mixture was cooled to $\sim 110^{\circ} \mathrm{C}$ with an air gun. $10 \mathrm{~mL}$ of toluene was then added and the mixture was allowed to return to room temperature. $5 \mathrm{~mL}$ of hexane was added and the mixture was centrifuged at $2147 \mathrm{~g}$ (4000 rpm) for $5 \mathrm{~min}$. The precipitate was redispersed in a mixture of $10 \mathrm{~mL}$ of hexane and $1 \mathrm{~mL}$ of oleic acid by sonication for $5 \mathrm{~min}$. The mixture was then centrifuged at $8586 \mathrm{~g}(8000 \mathrm{rpm})$ for $5 \mathrm{~min}$. The supernatant was separated. Since the supernatant in this case often contains a significant proportion of undesired smaller sizes and larger crystallites, special care must be taken to ensure efficient separation. $\sim 20 \mathrm{~mL}$ of methyl acetate was added in small quantities with continuous stirring to the supernatant until the solution became slightly turbid. It was then centrifuged at $8586 \mathrm{~g}(8000 \mathrm{rpm})$ for $5 \mathrm{~min}$. The precipitate was discarded. The supernatant was retained, and $\sim 4 \mathrm{~mL}$ of methyl acetate was added to it. This mixture was again centrifuged at $8586 \mathrm{~g}(8000 \mathrm{rpm})$ for $5 \mathrm{~min}$. The supernatant was discarded, and the precipitate containing the 554 species was redispersed in $3 \mathrm{~mL}$ of hexane. $10 \mathrm{~mL}$ of methyl acetate was again added, and centrifugation was repeated at $8586 \mathrm{~g}(8000 \mathrm{rpm})$ for $5 \mathrm{~min}$. The final product (precipitate) was stored in $2 \mathrm{~mL}$ of hexane.

\section{S1.2 Growth of isolated MSNCs}

MSNC 434 was synthesized as described above. The sample was diluted with hexane to have an optical density at the lowest energy excitonic transition $(434 \mathrm{~nm})$ equivalent to $\sim 20$ for a $1-\mathrm{cm}$ path length. 


\section{With solvent and ligands}

$6 \mathrm{~mL}$ of MSNC 434 dispersion was mixed with excess methyl acetate $(\sim 25 \mathrm{~mL})$ and centrifuged at $7547 \mathrm{~g}(7500 \mathrm{rpm})$ for $10 \mathrm{~min}$. The precipitate was redispersed in ODE and transferred to a three-neck round-bottomed flask. $68 \mathrm{mg}$ of cadmium myristate was added and the mixture was degassed under vacuum at $110{ }^{\circ} \mathrm{C}$. After $30 \mathrm{~min}$, the system was flushed with $\mathrm{N}_{2}$, and the temperature was raised to $180{ }^{\circ} \mathrm{C}$ to carry out the growth of the MSNCs. Aliquots were taken at regular intervals to monitor the growth using absorption spectroscopy.

\section{With ligands only}

10 vials were each filled with $0.5 \mathrm{~mL}$ of MSNC 434 dispersion. $2.5 \mathrm{~mL}$ of methyl acetate was then added to each vial. The mixtures were centrifuged to precipitate out the magic-sized nanocrystals. $6 \mathrm{mg}$ of cadmium myristate was added to each vial, which were subsequently placed in a pre-heated muffle furnace at $180^{\circ} \mathrm{C}$ under $\mathrm{N}_{2}$. The growth was monitored by removing a vial at a series of time points. $1 \mathrm{~mL}$ of hexane and $100 \mu \mathrm{L}$ of oleic acid were added to the cooled vials. They were sonicated for $\sim 5 \mathrm{~min}$ to redisperse the MSNCs for further analysis.

\section{With solvent only}

$6 \mathrm{~mL}$ of MSNC 434 dispersion was mixed with excess methyl acetate $(\sim 25 \mathrm{~mL})$ and centrifuged at $7547 \mathrm{~g}(7500 \mathrm{rpm})$ for $10 \mathrm{~min}$. The precipitate was redispersed in ODE and transferred to a three-neck round-bottomed flask. The dispersion was degassed under vacuum at $110{ }^{\circ} \mathrm{C}$. After $30 \mathrm{~min}$, the system was flushed with $\mathrm{N}_{2}$, and the temperature was raised to $180^{\circ} \mathrm{C}$ to carry out the growth of the MSNCs. Aliquots were taken at regular intervals to monitor the growth.

\section{S1.3 Characterization methods}

\section{Absorption spectroscopy}

MSNCs were characterized using a Varian Cary Scan 50 ultraviolet-visible (UV-vis) spectrophotometer. The wavelength range from $300-800 \mathrm{~nm}$ was analyzed. The aliquots were measured by diluting them in $2 \mathrm{~mL}$ of hexane, $100 \mu \mathrm{L}$ of oleic acid, and $20 \mu \mathrm{L}$ of ethanol (dilution factor of 20 ). The samples were measured using a 1 -cm path-length quartz cuvette.

\section{Photoluminescence (PL) and photoluminescence excitation (PLE) spectroscopy}

The PL and PLE spectra were recorded at room temperature $\left(\sim 23^{\circ} \mathrm{C}\right)$ using an Edinburgh Instruments FLS 980 photoluminescence spectrometer. The samples were measured using a 1-cm path-length quartz cuvette by diluting them in hexane to an optical density of 0.1 at the lowest energy exciton transition.

\section{Photoluminescence quantum yield (PLQY)}

PLQY was measured at room temperature using a Hamamatsu C11347 Quantaurus-QY spectrometer equipped with an integrating sphere. The excitation wavelength was $400 \mathrm{~nm}$. Purified MSNC samples 
were diluted in hexane and measured in quartz cuvettes with a 1-cm path length. The cuvettes were cleaned in a $1 \%$ solution of Hellmanex ${ }^{\mathrm{TM}}$ III after each sample to ensure accurate results.

\section{Powder X-ray diffraction (XRD)}

The XRD spectra were recorded on a Bruker D8 Advance instrument $(40 \mathrm{kV}, 40 \mathrm{~mA}$, $\lambda_{\text {CuK } \alpha}=0.15418 \mathrm{~nm}$ ). The samples for XRD were prepared by drop-casting concentrated hexane dispersions of MSNCs onto zero-background Si sample holders.

\section{Transmission electron microscopy (TEM)}

Transmission electron microscopy (TEM) micrographs were recorded on a JEOL JEM-1400 Plus, operated at $120 \mathrm{kV}$, or an FEI Talos F200X, operated at $200 \mathrm{kV}$. The scanning transmission electron microscopy (STEM) micrographs were recorded at $200 \mathrm{kV}$ on an FEI Talos F200X fitted with a highangle annular dark-field (HAADF) detector. The samples were prepared by drop-casting hexane dispersions of MSNCs (optical density of $\sim 0.6$ at the lowest energy excitonic transition for a 1-cm path length) onto 6-nm-thick carbon films supported by 200-mesh copper grids. It is important to note that damage is induced by the electron beam (Figure S7). To minimize this, microscope alignment was carried out at a particular sample location. However, images were recorded rapidly just outside this location.

\section{Size analysis}

For the size analysis, raw 1024×1024 pixel HAADF-STEM images were evaluated. While high magnifications can give a more precise particle-size estimation, it comes at the cost of accuracy due to beam damage (Figure S7). In contrast, low magnifications and large spot sizes minimize beam damage and give more accurate measurements. However, pixel discretization of the detector ultimately limits the precision. For the electron microscope used, this was optimized by choosing a magnification of 450,000X. All particle batches were imaged under identical operating conditions to keep other unknown errors systematic. Nevertheless, uncontrollable parameters during the synthesis, sample preparation, and imaging cannot be completely avoided, introducing random errors that can potentially adversely affect both precision and accuracy of the particle-size estimation. To minimize bias, the images were analyzed with a custom Python script. First a bandpass filter was applied in the Fourier-transformed image to eliminate high-frequency noise and reduce intensity variations in the background (lowfrequency noise). The minimum and maximum features for the bandpass filter were set to 1 and $12 \mathrm{~nm}$, respectively. Then, 48 pixels were cropped from each edge, to exclude edge effects of the electron beam. A binary Otsu threshold was applied to separate the objects (here MSNCs) from the background. Detected objects that touch an image border or have an area that is smaller than $1 \mathrm{~nm}^{2}$ were ignored for further analyses. The area was calculated by the number of pixels the object spanned. The effective diameters $(d)$ were extracted by assuming the areas of the detected objects $(A)$ to be equal to a circle of same area, i.e. $d=\sqrt{4 A / \pi}$. 


\section{Nuclear magnetic resonance spectroscopy (NMR)}

All spectra were collected on a Bruker Ascend Aeon 400-MHz spectrometer. ${ }^{1} \mathrm{H}-\mathrm{NMR},{ }^{13} \mathrm{C}-\mathrm{NMR}$, and

${ }^{77} \mathrm{Se}-\mathrm{NMR}$ spectra were recorded with predefined pulse programs. The samples for NMR were prepared by dispersing MSNCs in $\mathrm{CDCl}_{3}$.

\section{S1.4 Comments on the sizes extracted from transmission electron microscopy (TEM)}

Using the procedure outlined in S1.3, we extracted the average sizes of our MSNCs from TEM images (see Figure S6). For the 434, 455, 476, and 494 species, we obtained sizes of 2.13, 2.36, 2.55, and $2.69 \mathrm{~nm}$, respectively. We stress that these values represent effective diameters. In other words, we determine the particle cross-sectional area, convert that area to a circle, and extract its diameter. For the series of particles in Figure S6, the size increment is $0.23,0.19$, and $0.14 \mathrm{~nm}$, respectively, which yields an average increase in size between MSNCs of $0.19 \pm 0.05 \mathrm{~nm}$.

It is useful to consider what type of increase in size between MSNCs would be consistent with our model. If we assume that the particles are perfect tetrahedra, an additional monolayer of CdSe added to a $\{111\}$ facet has a thickness of $0.351 \mathrm{~nm}$. If one then considers the two-dimensional projection (cross section) of such a tetrahedron, one would expect an increase in effective diameter of $\sim 0.31-0.35 \mathrm{~nm}$, depending on which projection is considered. This value is clearly higher than the $0.19 \mathrm{~nm}$ observed in experiment. A possible explanation for this discrepancy is that the particles that are imaged are truncated tetrahedra. The absence of the vertices can reduce the expected increment in effective diameter between MSNCs to $\sim 0.20 \mathrm{~nm}$, in line with our experimental results. Similarly, the vertices may be present, but poorly imaged, yielding a similar result. Further imaging experiments are needed to clarify the particle shape.

\section{S2 NUCLEATION THEORY APPLIED TO MSNCS}

\section{S2.1 Geometric description of MSNC}

XRD measurements of MSNCs reveal that they are crystalline with a zinc-blende lattice. In line with previous reports, ${ }^{\mathrm{S} 3, \mathrm{~S} 4}$ we assume that their shape is tetrahedral and the exposed nanocrystal facets are Cd-terminated $\{111\}$ surfaces. Our model uses the geometric properties of tetrahedra to facilitate the description of MSNCs. The lateral number of monomers $(n)$ (see Figure $3 \mathrm{a}$ in the main text) defines all geometric quantities needed in our model: the total number of monomers on an edge, on a surface facet, and in a MSNC. The number of monomers on an edge is $n$, the number of monomers on a MSNC facet is given by the triangular number $[\Gamma(n)]$, and the total number of monomers in a MSNC is given by the tetrahedral number $[\Phi(n)]$. The tetrahedral and triangular number, together with related properties, are defined below:

$$
\Phi(n)=\sum_{i=1}^{n} \Gamma(i)=\frac{n(n+1)(n+2)}{6} ; \frac{d \Phi}{d n}=\frac{3 n^{2}+6 n+2}{6},
$$




$$
\begin{gathered}
\Gamma(n)=\sum_{i=1}^{n} i=\frac{n(n+1)}{2} ; \frac{d \Gamma}{d n}=\frac{2 n+1}{2}, \\
\Phi(n)=\Phi(n-1)+\Gamma(n) .
\end{gathered}
$$

The definition provided in eq S3 links the tetrahedral and triangular numbers. It has a straightforward physical interpretation: covering one of the four identical facets of a tetrahedron [lateral size $(n-1)$ ] with a triangular surface (lateral size $n$ ), yields the next-larger tetrahedron (lateral size $n$ ).

\section{S2.2 Energy of forming 3D nuclei and 2D surface islands}

As discussed in the main text, we describe the energy of forming a 3D nucleus using classical nucleation theory. For a tetrahedral-shaped 3D nucleus with $n$ monomers on its side, the energy of formation is given as:

$$
\Delta G^{3 \mathrm{D}}(n)=-\Phi(n) \Delta \mu+4 \varepsilon_{A} \Gamma(n),
$$

where $\Delta \mu$ is the supersaturation, i.e. the difference in chemical potential between a monomer in the solution and in the bulk crystal. The energy of breaking a crystalline bond and passivating it with a ligand is described by $\varepsilon_{A}$. It relates to the surface energy $\left(\sigma_{A}\right)$ as $\varepsilon_{A}=\sigma_{A} \rho_{A}$, where $\rho_{A}$ is the surface area per dangling bond. Monomers located within the surface exhibit one dangling bond, while monomers at the edges or vertices of MSNCs exhibit two and three dangling bonds, respectively. Each monomer at these sites exhibits exactly one dangling bond per surface that includes them. Therefore, $4 \Gamma(n)$ is the total number of dangling bonds per MSNC of size $n$. For simplicity, we will refer to $\varepsilon_{A}$ as the surface energy.

The energy of forming a 2D island growing on the surface of a MSNC is given as:

$$
\Delta G^{2 \mathrm{D}}(m)=\left\{\begin{array}{cc}
-\Gamma(m) \Delta \mu+4 \varepsilon_{A} m+\varepsilon_{S} m & \text { if } m<n+1 \\
-\Gamma(m) \Delta \mu+4 \varepsilon_{A} m & \text { if } m=n+1
\end{array} .\right.
$$

Here, $m$ is the lateral number of monomers forming the 2D surface island (see Figure $3 \mathrm{a}$ in the main text). The step energy, $\varepsilon_{S}$, accounts for the fact that ligand passivation at the step front is different, compared to other surface sites. When the facet of a MSNC is completed, we need to account for the disappearance of the step front. For an MSNC of size $n$, this occurs when a 2D surface island of size $m=n+1$ is formed.

\section{S2.3 Effects of changes in supersaturation}

In the main text, the stability of different-sized MSNCs as well as the transition states that link them, is plotted for fixed supersaturation (see Figure $3 \mathrm{~d}$ in the main text). However, as nucleation and growth proceed, the monomer concentration in the melt decreases, thus decreasing the supersaturation. Here, we aim to provide a detailed derivation of how changes in supersaturation affect nucleation and growth. In Figure S11 we plot the overall energy of formation curve for forming MSNCs at different 
supersaturations. As the supersaturation decreases, energy barriers linking different-sized MSNCs increase. Further, the critical size for forming a 3D nucleus increases. This means that the threshold size for MSNC stability increases. Finally, the size range of MSNCs exhibiting size-dependent barriers shifts to larger sizes.

We wish to establish the link between the critical size of forming a 3D nucleus $\left(n_{\text {crit }}^{3 \mathrm{D}}\right)$ and the critical supersaturation $\left[\Delta \mu_{\mathrm{crit}}^{3 \mathrm{D}}(n)\right]$ at which a $3 \mathrm{D}$ nucleus of lateral size $n$ dissolves. The critical size of forming a $3 \mathrm{D}$ nucleus $\left(n_{\text {crit }}^{3 \mathrm{D}}\right)$ is defined as:

$$
\left.\frac{\partial \Delta G^{3 \mathrm{D}}}{\partial n}\right|_{n_{\text {crit }}^{3 \mathrm{D}}}=\left.\left[-\left(\frac{3 n^{2}+6 n+2}{6}\right) \Delta \mu+4 \varepsilon_{A}\left(\frac{2 n+1}{2}\right)\right]\right|_{n_{\text {crit }}^{3 \mathrm{D}}}=0
$$

Eq S6 describes how the 3D critical size $\left(n_{\text {crit }}^{3 \mathrm{D}}\right)$ depends on supersaturation or, vice versa, it defines the critical supersaturation $\left[\Delta \mu_{n, \text { crit }}^{3 \mathrm{D}}\right]$ below which a MSNC of given size $(n)$ dissolves:

$$
\Delta \mu_{n, \mathrm{crit}}^{3 \mathrm{D}}=\frac{12 \varepsilon_{A}(2 n+1)}{3 n^{2}+6 n+2} .
$$

From eq S7 we recognize that larger MSNCs exhibit a smaller 3D critical supersaturation. In turn, as the supersaturation decreases, $n_{\text {crit }}^{3 \mathrm{D}}$ increases.

We follow a similar procedure to determine how the size range of MSNCs that exhibit size-dependent barriers varies with supersaturation. At fixed supersaturation, the energy of growing on a bulk facet is linked to a $2 \mathrm{D}$ island with a lateral number of monomers, $m_{\text {crit }}^{2 \mathrm{D}}$. Because $m_{\text {crit }}^{2 \mathrm{D}}$ determines the growth barrier on a bulk facet $\left[\Delta G^{2 \mathrm{D}}\left(m_{\mathrm{crit}}^{2 \mathrm{D}}\right)\right]$, the barriers involved in growing $2 \mathrm{D}$ islands with a lateral number of monomers larger or equal to $m_{\text {crit }}^{2 \mathrm{D}}$ are identical to that of the bulk, i.e. size independent. In contrast, the barriers for growing 2D islands with a lateral number of monomers smaller than $m_{\text {crit }}^{2 \mathrm{D}}$ are size dependent $\left[\Delta G^{2 \mathrm{D}}(m)\right]$ and smaller than the bulk barrier $\left[\Delta G^{2 \mathrm{D}}\left(m_{\text {crit }}^{2 \mathrm{D}}\right)\right]$. The 2D critical island size $m_{\text {crit }}^{2 \mathrm{D}}$ is then given by:

$$
\left.\frac{\partial \Delta G^{2 \mathrm{D}}}{\partial m}\right|_{m_{\text {crit }}^{2 \mathrm{D}}}=\left.\left[-\left(\frac{2 m+1}{2}\right) \Delta \mu+\left(4 \varepsilon_{A}+\varepsilon_{S}\right)\right]\right|_{m_{\text {crit }}^{2 \mathrm{D}}}=0
$$

At fixed supersaturation, eq S8 defines the size-range of 2D islands exhibiting either size-dependent ( $m<m_{\text {crit }}^{2 \mathrm{D}}$ ) or size-independent ( $m \geq m_{\text {crit }}^{2 \mathrm{D}}$ ) barriers. Conversely, eq S8 also defines the 2D critical supersaturation $\left(\Delta \mu_{m, \text { crit }}^{2 \mathrm{D}}\right)$ below which the barrier to grow 2D islands of lateral size $m$ is smaller than the barrier to grow on a bulk facet:

$$
\Delta \mu_{m, \mathrm{crit}}^{2 \mathrm{D}}=\frac{2\left(4 \varepsilon_{A}+\varepsilon_{S}\right)}{2 m+1}
$$

Like for the 3D critical size, the $2 \mathrm{D}$ critical size $\left(m_{\mathrm{crit}}^{2 \mathrm{D}}\right)$ and supersaturation $\left(\Delta \mu_{m, \text { crit }}^{2 \mathrm{D}}\right)$ are inversely proportional. 
Having defined the effect of supersaturation on the 2D and 3D critical size, we determine how the range of stable MSNCs exhibiting size-dependent barriers to the next-larger MSNC depends on supersaturation. We note that transitioning from MSNCs with $n$ lateral monomers to the subsequent MSNCs requires the formation of a $2 \mathrm{D}$ surface island of $(n+1)$ monomers in lateral size. The barrier to transition from one MSNC to the next will only be size dependent if the required 2D surface island is smaller than the $2 \mathrm{D}$ critical size. In terms of supersaturation, this translates to the following condition:

$$
\Delta \mu<\Delta \mu_{n+1, \text { crit }}^{2 \mathrm{D}}
$$

MSNCs with $n$ lateral monomers can only be stable and exhibit size-dependent barriers if the following inequality holds:

$$
\Delta \mu_{n, \text { crit }}^{3 \mathrm{D}}<\Delta \mu_{n+1, \text { crit }}^{2 \mathrm{D}}
$$

Then, a supersaturation regime exists where the MSNCs are stable $\left(\Delta \mu_{n, \text { crit }}^{3 \mathrm{D}}<\Delta \mu\right)$ and exhibit sizedependent barriers to transition to the next-larger size $\left(\Delta \mu<\Delta \mu_{n+1, \text { crit }}^{2 \mathrm{D}}\right)$. This regime is highlighted in beige for different-sized MSNCs in Figure $4 \mathrm{a}$ in the main text.

\section{S3 MODEL PARAMETERS}

The model presented above depends on two parameters only: the step energy $\left(\varepsilon_{S}\right)$ and the energy of breaking a crystalline bond and passivating it with a ligand $\left(\varepsilon_{A}\right)$. All calculations presented in the main text have been performed using $\varepsilon_{A}=128 \mathrm{meV}$ and $\varepsilon_{S}=5 \varepsilon_{A}$. In the following, we discuss how these parameters where chosen.

\section{S3.1 Surface energy $\left(\varepsilon_{A}\right)$}

The energy of breaking a crystalline bond and passivating it with a ligand $\left(\varepsilon_{A}\right)$ is tightly linked to the surface energy $\left(\sigma_{A}\right)$ of a passivated, Cd-terminated $\{111\}$ facet. Here, we first discuss $\sigma_{A}$ for unpassivated (bare) surfaces, and then incorporate the effect of ligands on $\sigma_{A}$.

Bare $\{111\}$ facets have one dangling bond per monomer. For CdSe, their surface energy was reported to be $46 \mathrm{meV} / \AA^{2}$ (Ko et al. ${ }^{\mathrm{S} 5}$ ) or $52 \mathrm{meV} / \AA^{2}$ (Liu et al. ${ }^{\mathrm{S} 6}$ ). Ligands passivate dangling bonds and therefore reduce the surface energy of bare facets. This stabilizing effect strongly depends on the ligand type, on how it binds to the surface, and the surface coverage. ${ }^{\mathrm{S} 5, \mathrm{S7}}$ For passivated surfaces, $\sigma_{A}$ is defined as: ${ }^{\text {s5 }}$

$$
\sigma_{A, p}=\sigma_{A, b}-\Delta \sigma_{p}
$$

where $\sigma_{A, p}$ and $\sigma_{A, b}$ are the surface energies of the passivated $\left(\sigma_{A, p}\right)$ and bare $\left(\sigma_{A, b}\right)$ surfaces, and $\Delta \sigma_{p}$ quantifies the stabilizing effect of the passivating ligands. For Cd-terminated $\{111\}$ facets passivated with acetate, Ko et al. ${ }^{\mathrm{S} 5}$ report $\Delta \sigma_{p}$ to be $38 \mathrm{meV} / \AA^{2}$ respectively. The surface energy of the passivated 
facet is then $8 \mathrm{meV} / \AA^{2}$. Using $16 \AA^{2}$ for the surface area per dangling bond $\left(\rho_{A}\right)$ yields $128 \mathrm{meV}$ for $\varepsilon_{A}$, which is the value used in all of our calculations.

\section{S3.2 Step energy $\left(\varepsilon_{S}\right)$}

In our model, the step energy $\left(\varepsilon_{S}\right)$ accounts for the fact that monomers located on the step edge cost an additional energy compared to monomers located on a terminated facet. This can occur because ligand passivation at the step front is different (i.e. less effective) compared to terminated facets, as proposed for CdSe nanoplatelets (NPLs). ${ }^{\mathrm{S} 8}$ However, NPLs have Cd-rich $\{100\}$ facets exposed, which is different compared to MSNCs, which have $\{111\}$ facets exposed.

For $\{111\}$ facets, no data on the step energy exists. In the absence of data, we determine $\varepsilon_{S}$ (unknown) from $\varepsilon_{A}$ (known) by imposing that $\Delta \mu_{n \text {,crit }}^{3 \mathrm{D}}<\Delta \mu_{n+1 \text {,crit }}^{2 \mathrm{D}}$ holds for all MSNC sizes $n$. From this inequality we obtain the following condition on $\varepsilon_{S}$ :

$$
\varepsilon_{S}>\varepsilon_{A}\left(\frac{6(2 n+3)(2 n+1)}{3 n^{2}+6 n+2}-4\right)
$$

For large $n, \varepsilon_{S}$ is exactly four times larger than $\varepsilon_{A}$, while for $n=2, \varepsilon_{S}$ needs to be greater than $4.077 \varepsilon_{A}$. For the calculations reported in the main text, we chose $\varepsilon_{S}=5 \varepsilon_{A}$ to fulfill eq S13. Note that when $\varepsilon_{S}$ is chosen such that that eq S13 is not fulfilled, no size regime of stable MSNCs exhibiting size-dependent barriers exists (see Figure S12). This will affect MSNC growth. However, we note that MSNCs are still local minima in the energy of formation curve.

\section{S4 POPULATION-BALANCE MODEL}

As MSNCs grow they incorporate monomers, which in turn reduces the monomer concentration in solution, $c_{1}$. The monomer concentration in solution is linked to the supersaturation as $\Delta \mu=k_{B} T \ln \left(c_{1} / c_{\infty}^{*}\right)$, where $c_{\infty}^{*}$ is the monomer concentration at the bulk solubility. In the main text we refer to $c_{1} / c_{\infty}^{*}$ as $\tilde{c}_{1}$. Intuitively, changes in $\tilde{c}_{1}$ depend on the growth and dissolution of MSNCs. At the same time, changes in $\tilde{c}_{1}$-and therefore also $\Delta \mu$-affect the growth rates of different MSNC populations, because the barriers to transition from one MSNC to the next-larger size vary (Figure $4 \mathrm{~b}$ in the main text).

To include all these effects and simulate the temporal evolution of different MSNCs, we use a population-balance model. Experiments suggest that growth and dissolution occur layer by layer through the addition or removal of one monolayer. Therefore, we assume that each MSNC size can either grow to the next-larger MSNC (consuming monomers) or dissolve to the next-smaller MSNC (generating monomers). As a result, we describe the temporal change in concentration $\left(c_{n}\right)$ of MSNC species of size $n \geq 2$ as:

$$
\frac{d \tilde{c}_{n}}{d t}=I_{n-1} \tilde{c}_{n-1}+D_{n+1} \tilde{c}_{n+1}-\left(I_{n}+D_{n}\right) \tilde{c}_{n}
$$


here given for the normalized concentrations $\tilde{c}_{n}=c_{n} / c_{\infty}^{*}$. Each of the four fluxes entering the righthand side of eq S14 are given by both a concentration and a rate. The growth rate $\left(I_{i}\right)$ depends on the supersaturation, the surface and step energies $\left(\varepsilon_{S}, \varepsilon_{A}\right)$, the temperature $(T)$, as well as the attempt frequency $C$. We define it as:

$$
I_{n}=C \exp \left[-\frac{E_{n, \text { barr }}}{k_{B} T}\right] ; E_{n, \text { barr }}=\left\{\begin{array}{cl}
\Delta G^{2 \mathrm{D}}(n+1)+\varepsilon_{S}(n+1) & \text { if } n+1 \leq m_{\text {crit }}^{2 \mathrm{D}} \\
\Delta G^{2 \mathrm{D}}\left(m_{\text {crit }}^{2 \mathrm{D}}\right) & \text { if } n+1>m_{\text {crit }}^{2 \mathrm{D}}
\end{array} .\right.
$$

We distinguish two cases in eq S15 because MSNCs that have to grow facets smaller than the 2D critical island size $\left(m_{\text {crit }}^{2 \mathrm{D}}\right)$ exhibit size-dependent barriers to transition to the next-larger MSNCs. To correctly compute the barriers $\left(E_{n, \text { barr }}\right)$, we need to explicitly include the step-energy term $\left[\varepsilon_{S}(n+1)\right]$ for $n+1 \leq m_{\text {crit }}^{2 \mathrm{D}}$. Otherwise, this term would be missing due to the definition of $\Delta G^{2 \mathrm{D}}(n+1)$ in eq S5. The dissolution rates $\left(D_{i}\right)$ are defined by imposing the equilibrium condition. Namely, at the critical supersaturation $\Delta \mu_{n, \text { crit }}^{3 \mathrm{D}}$, the rate of forming a MSNC of lateral size $n\left(I_{n-1}\right)$ has to be the same as the rate of dissolution $\left(D_{n}\right)$ :

$$
D_{n}=I_{n-1}\left(\Delta \mu_{n, \text { crit }}^{3 \mathrm{D}}\right)
$$

Finally, temporal changes in the monomer concentration in solution, $\tilde{c}_{1}$, are accounted for by imposing mass balance on the overall system. In differential form this reads as:

$$
\frac{d \tilde{c}_{1}}{d t}=-\sum_{n \geq 2}^{N} \Phi(n) \frac{d \tilde{c}_{n}}{d t},
$$

where $N$ is the largest size considered. In principle, $N$ should be infinite. In practice, $N$ has to be finite. In our simulations it is chosen to be 30 , which is large enough not to have any effect on the growth dynamics. For the largest size considered, we neglect the $D_{N+1} \tilde{c}_{N+1}$ term in eq S14.

The master equations (eq S14 and S17) determine the temporal evolution of our system. They constitute a system of non-linear ordinary differential equations, which we solve using the highperformance Python compiler Numba. In all our simulations we assume the temperature to be $200{ }^{\circ} \mathrm{C}$, and an attempt frequency of $1 \times 10^{17}$. The attempt frequency which has units of $\mathrm{s}^{-1}$ defines the normalized time $\tau=C t$. For the initial supersaturation in our simulations we assumed a value of $550 \mathrm{meV}$ (which is larger than $\Delta \mu_{2, \text { crit }}^{3 \mathrm{D}}$ ). Increasing the initial supersaturation reduces the times at which small sizes $(n \leq 4)$ are observed. However, we find that it has no substantial impact on the overall MSNC growth dynamics. 


\section{S5 SUPPORTING FIGURES}
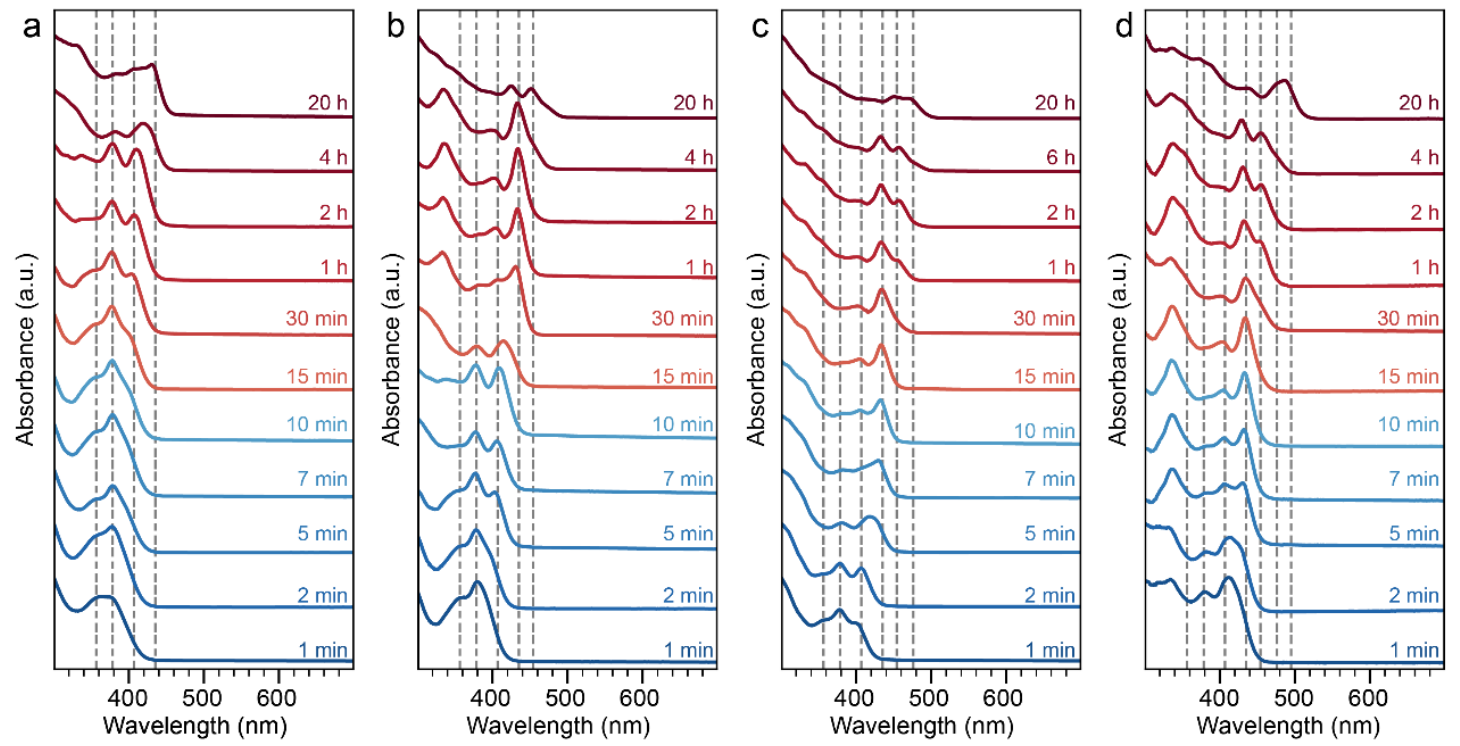

Figure S1. Temporal evolution of optical absorption spectra of CdSe MSNCs grown at different temperatures: (a) $120^{\circ} \mathrm{C}$, (b) $150{ }^{\circ} \mathrm{C}$, (c) $180^{\circ} \mathrm{C}$, and (d) $210{ }^{\circ} \mathrm{C}$. Each spectrum is labeled with the time after injection. The vertical dashed lines indicate the lowest energy excitonic peaks for MSNC product species (at 355, 380, 408, 434, 455, 476, 494, 508, and $523 \mathrm{~nm}$ ). As we increase the growth temperature, the observed range of discrete excitonic features shifts from shorter to longer wavelengths. 


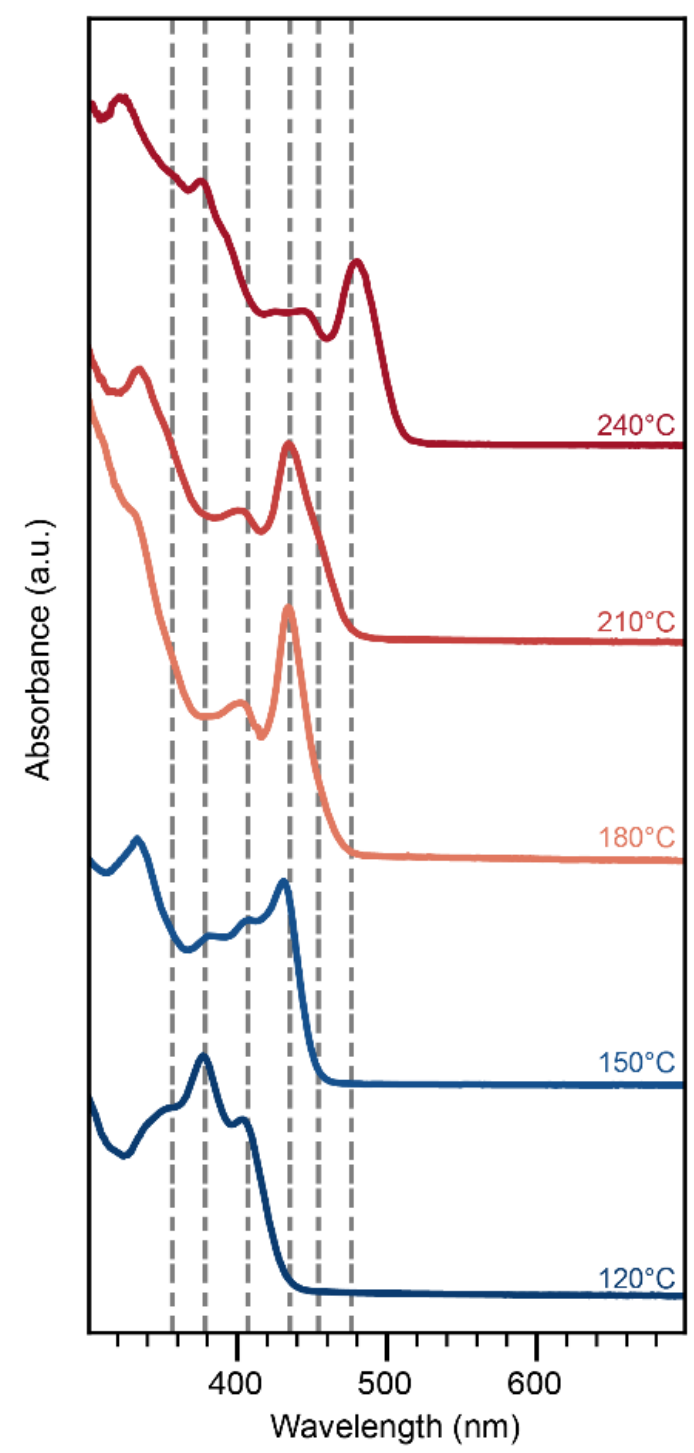

Figure S2. Effect of growth temperature on the synthesis of CdSe MSNCs. Optical absorption spectra of aliquots taken $30 \mathrm{~min}$ after the injection of bis(stearoyl) selenide at different temperatures. Each spectrum is labeled with the injection and growth temperature. Higher injection and growth temperatures result in faster discrete and sequential transitions to absorption features at longer wavelengths. The vertical dashed lines indicate the lowest energy excitonic peaks for six MSNC species (at $355,380,408,434,455$, and $476 \mathrm{~nm}$ ). 


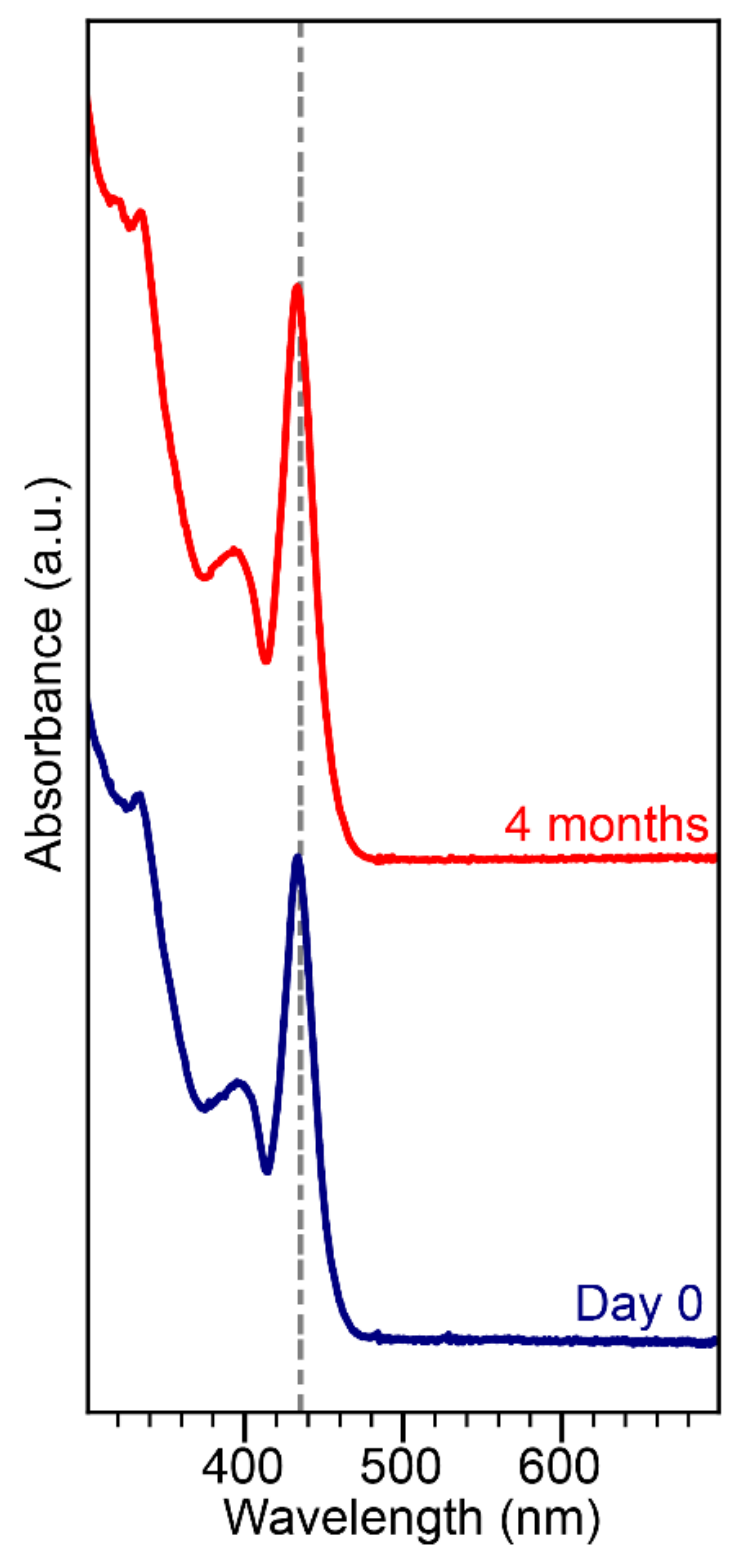

Figure S3. Stability of CdSe MSNCs under ambient conditions. Optical absorption spectra of isolated 434 immediately after the synthesis (blue) and after 4 months (red). The products were stored under ambient conditions as a liquid dispersion in hexane (optical density at the lowest energy excitonic transition $>1.5$ for a $1-\mathrm{cm}$ path length). 

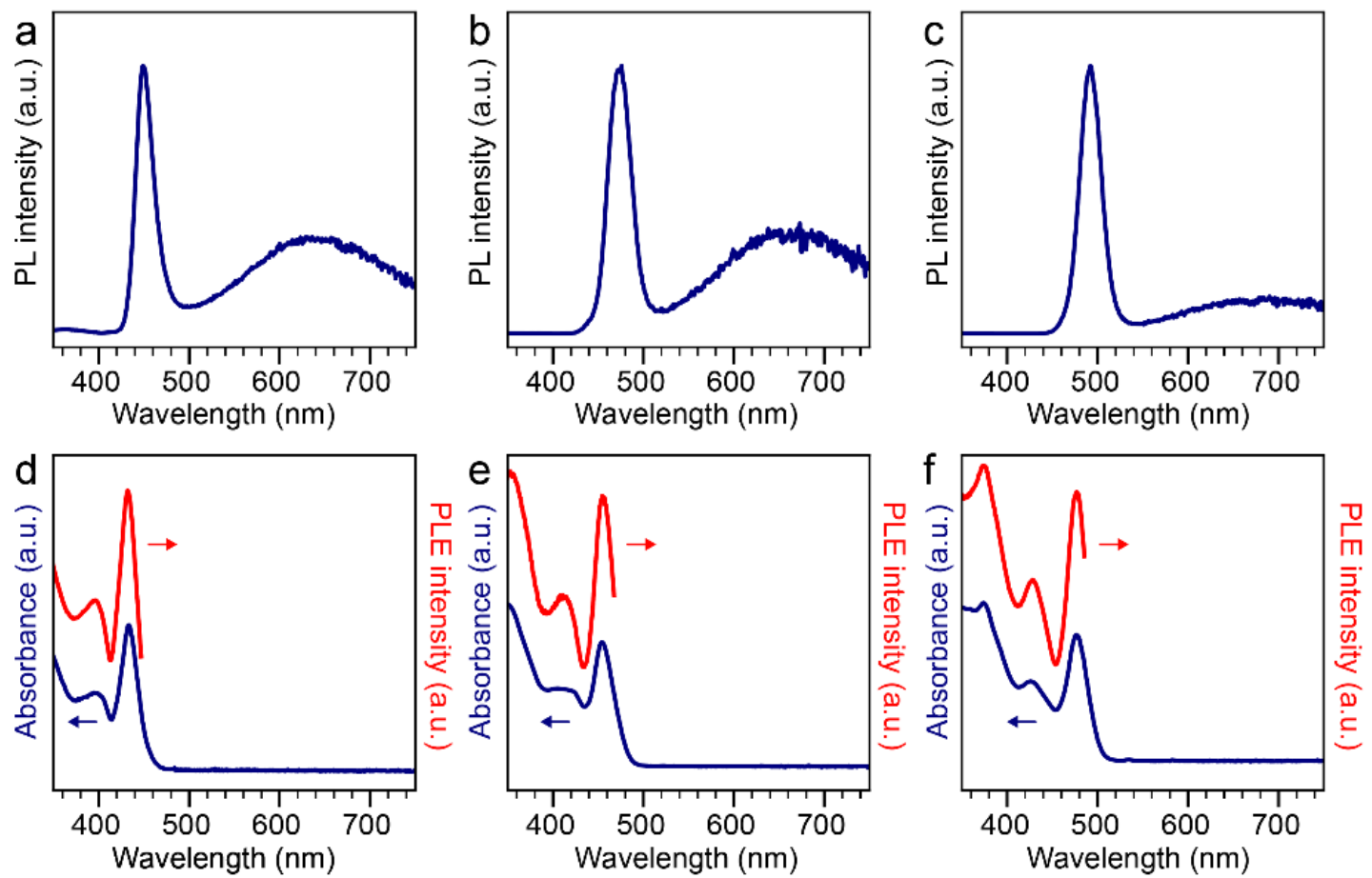

Figure S4. Optical characterization of isolated CdSe MSNCs. Room-temperature photoluminescence (PL) spectra of the isolated (a) 434, (b) 455, and (c) 476 species. For PL, the excitation wavelength is $300 \mathrm{~nm}$. The observation of only single PL peaks at 450, 474, and $492 \mathrm{~nm}$, respectively, confirms successful isolation of each species. Room-temperature photoluminescence excitation (PLE, red) and absorption (blue) spectra of the isolated (d) 434, (e) 455, and (f) 476 species. For PLE, the emission is monitored at 452, 472, and $490 \mathrm{~nm}$, respectively. The strong similarity between the absorption and PLE spectra in each case indicates that the higher-energy absorption features are due to excited states of the isolated MSNC species. 

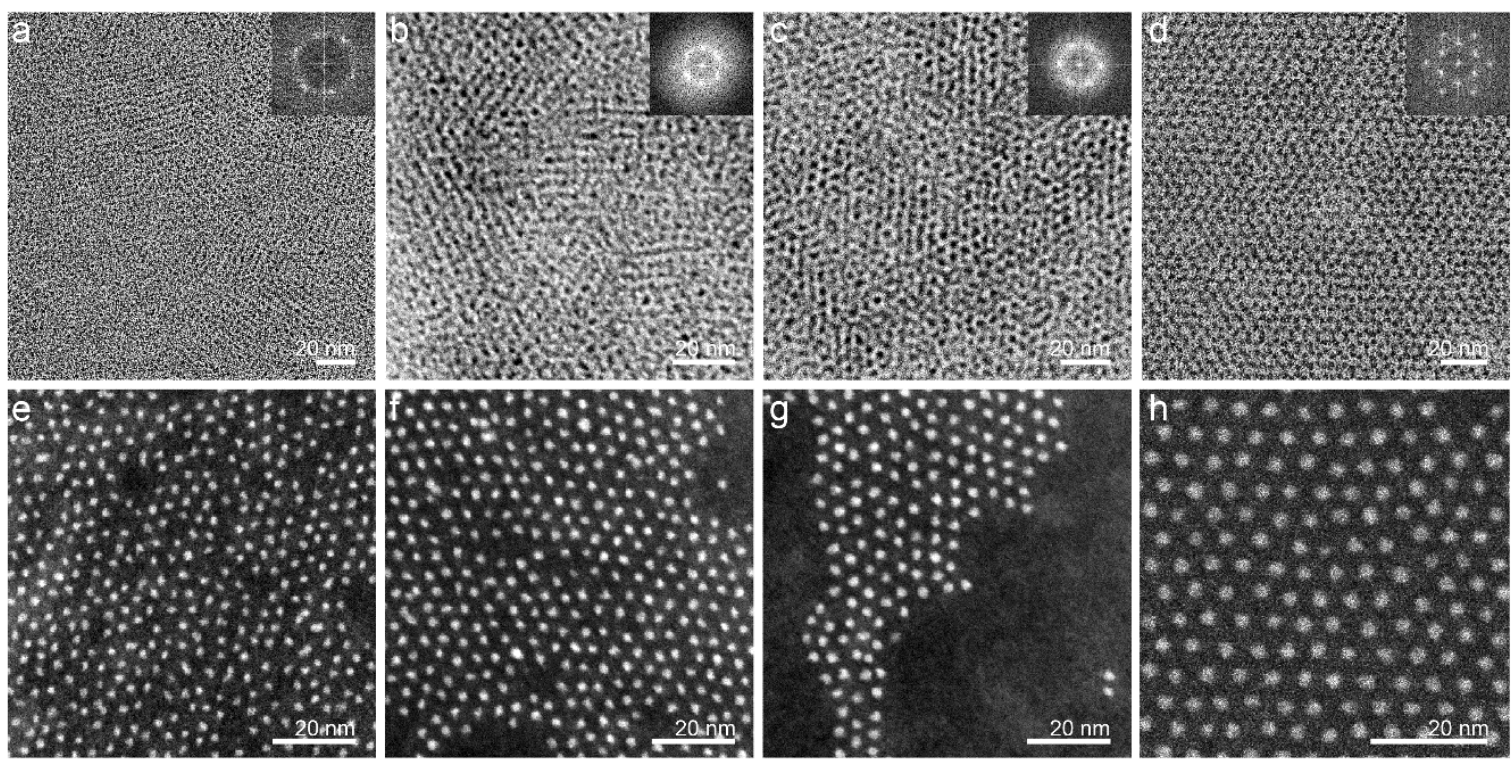

Figure S5. Electron microscopy images of isolated CdSe MSNC species. The transmission electron microscopy (TEM) images at lower magnification and the corresponding fast-Fourier transforms (FFT) (inset right) are shown for: (a) 434, (b) 455, (c) 476, and (d) 494 species. The formation of assemblies of MSNCs suggests uniform particles. High-angle annular dark-field (HAADF) scanning transmission electron microscopy (STEM) images of (e) 434, (f) 455, (g) 476, and (h) 494 species reveal no sign of molecular templates. While efforts were taken to reduce beam damage in these images (see section S1.3), the shape of the particles may have evolved during the exposure. 

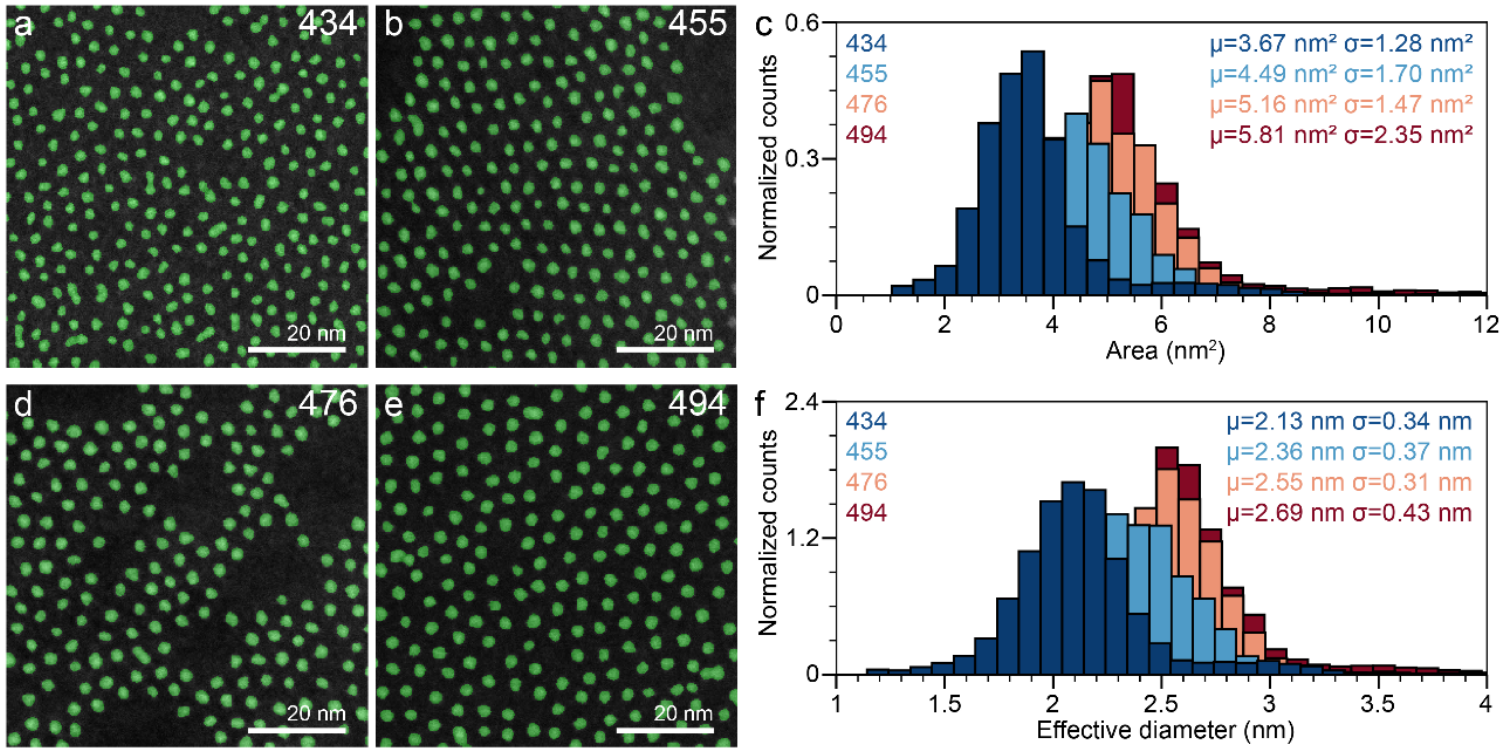

Figure S6. Size analysis of CdSe MSNCs using transmission electron microscopy. Images show detected objects (green) after evaluating the high-angle annular dark-field (HAADF) scanning transmission electron microscopy (STEM) images of (a) 434, (b) 455, (d) 476, and (e) 494 species using a custom Python script. (c) Normalized histograms with mean $(\mu)$ and standard deviation $(\sigma)$ of the area of the detected objects. (f) The extracted effective diameter. The bin sizes of histograms in (c) and (f) are $0.4 \mathrm{~nm}^{2}$ and $0.1 \mathrm{~nm}$, respectively. A total of 21,275 (434), 12,143 (455), 9,915 (476), and 14,767 (494) particles were analyzed. The mean areas and effective diameters increase, consistent with the discrete jumps observed in the optical spectra (see Figure 1a in the main text). We note that while strategies to minimize electron-beam damage in the STEM images were followed (see section S1.3), the images are still potentially affected. See also Figure S7. 

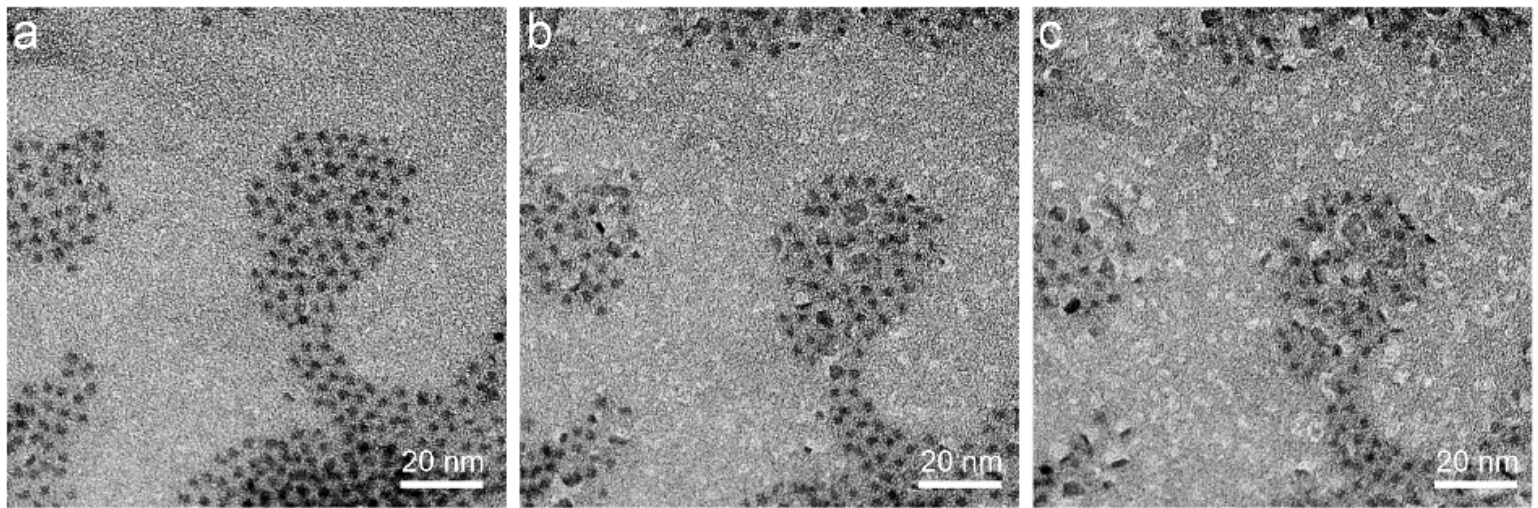

Figure S7. Shape evolution and damage induced by the electron beam for CdSe MSNCs. The transmission electron micrographs are at a magnification of 300k for isolated 494 . The sample was fixed at a particular position and exposed to the $120 \mathrm{kV}$ beam for a few minutes. The images were recorded at (a) $0 \mathrm{~min}$, (b) $2 \mathrm{~min}$, and (c) $4 \mathrm{~min}$. The drift induced at higher magnification slightly shifts the position of the sample over time. The rate of beam-induced damage increases at higher magnifications. 

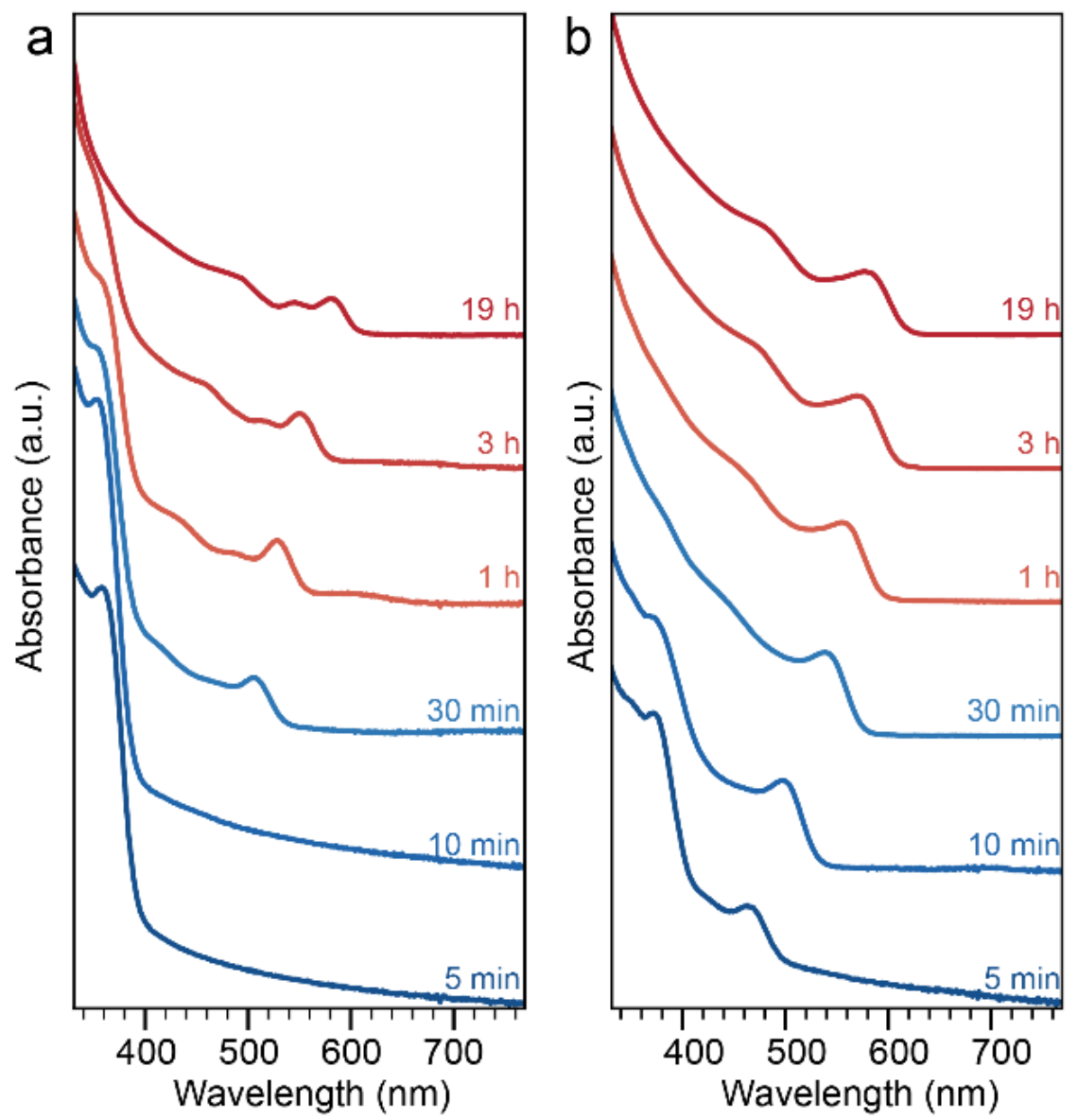

Figure S8. Replacing the reactive Se precursor in CdSe MSNC synthesis with less reactive precursors. Temporal evolution of optical absorption spectra when the Se precursor, bis(stearoyl) selenide, was replaced with equimolar (a) elemental selenium or (b) trioctylphosphine selenide. Each spectrum is labeled with the injection and growth time. The absence of discrete and sequential evolution of excitonic features suggests that magic-sized nanocrystals have not been formed. 


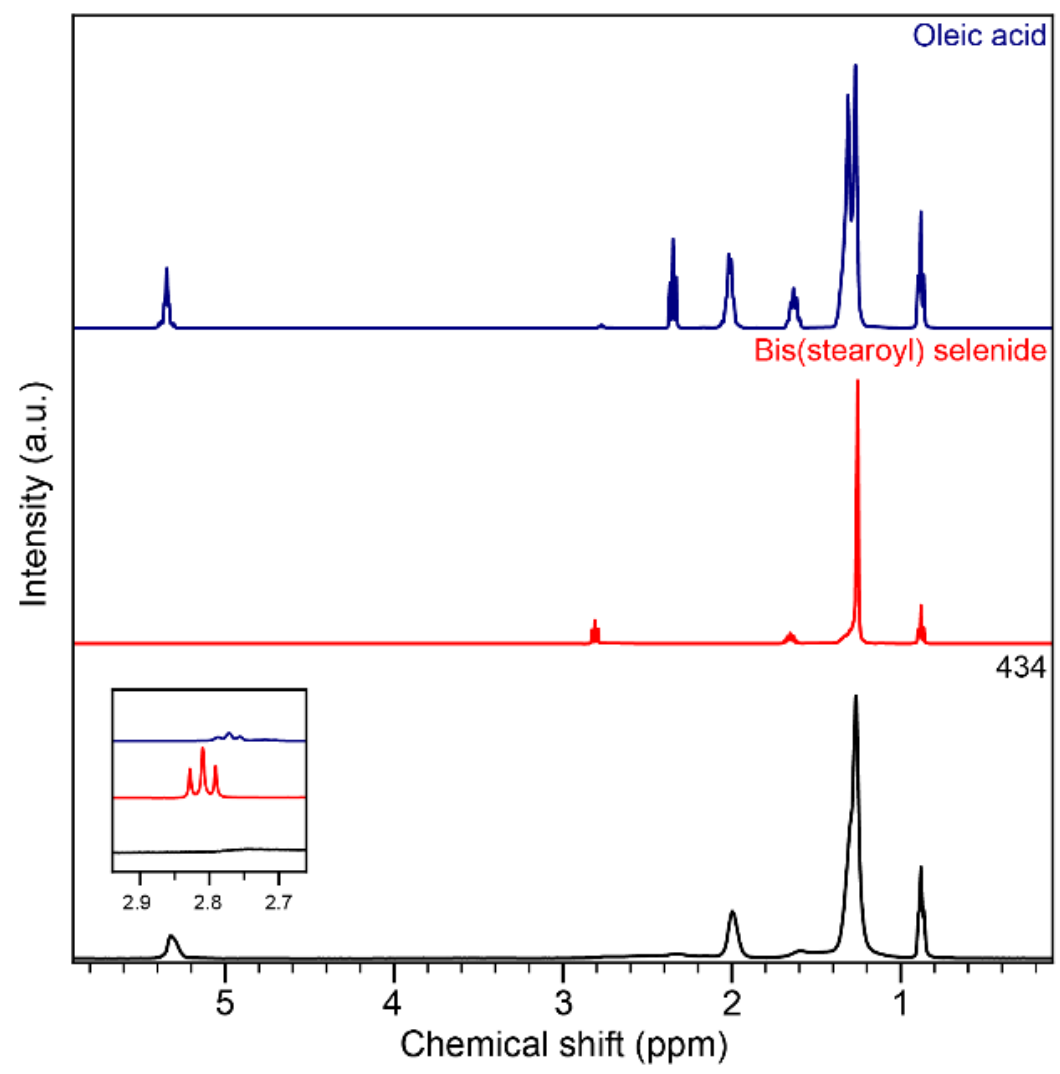

Figure S9. Nuclear magnetic resonance spectrum of isolated CdSe MSNC 434. ${ }^{1} \mathrm{H}$ NMR spectra (400 MHz) of isolated 434 (black), bis(stearoyl) selenide (red), and pure oleic acid (blue) in $\mathrm{CDCl}_{3}$. The broadness of the peaks in the spectrum of 434 is presumably due to the restricted movement of ligands when they are attached to the crystallite surface. The absence of a peak at $2.81 \mathrm{ppm}$ (inset) suggests the absence of the bis(stearoyl) selenide precursor in the isolated 434. Furthermore, the peaks of 434 were quantitatively integrated. The ratios of the intensities of the different peaks match the ratios in the oleic acid molecule. This supports the existence of only one molecule (the ligand) in the system ensuring complete isolation of MSNC from the selenium precursors. 


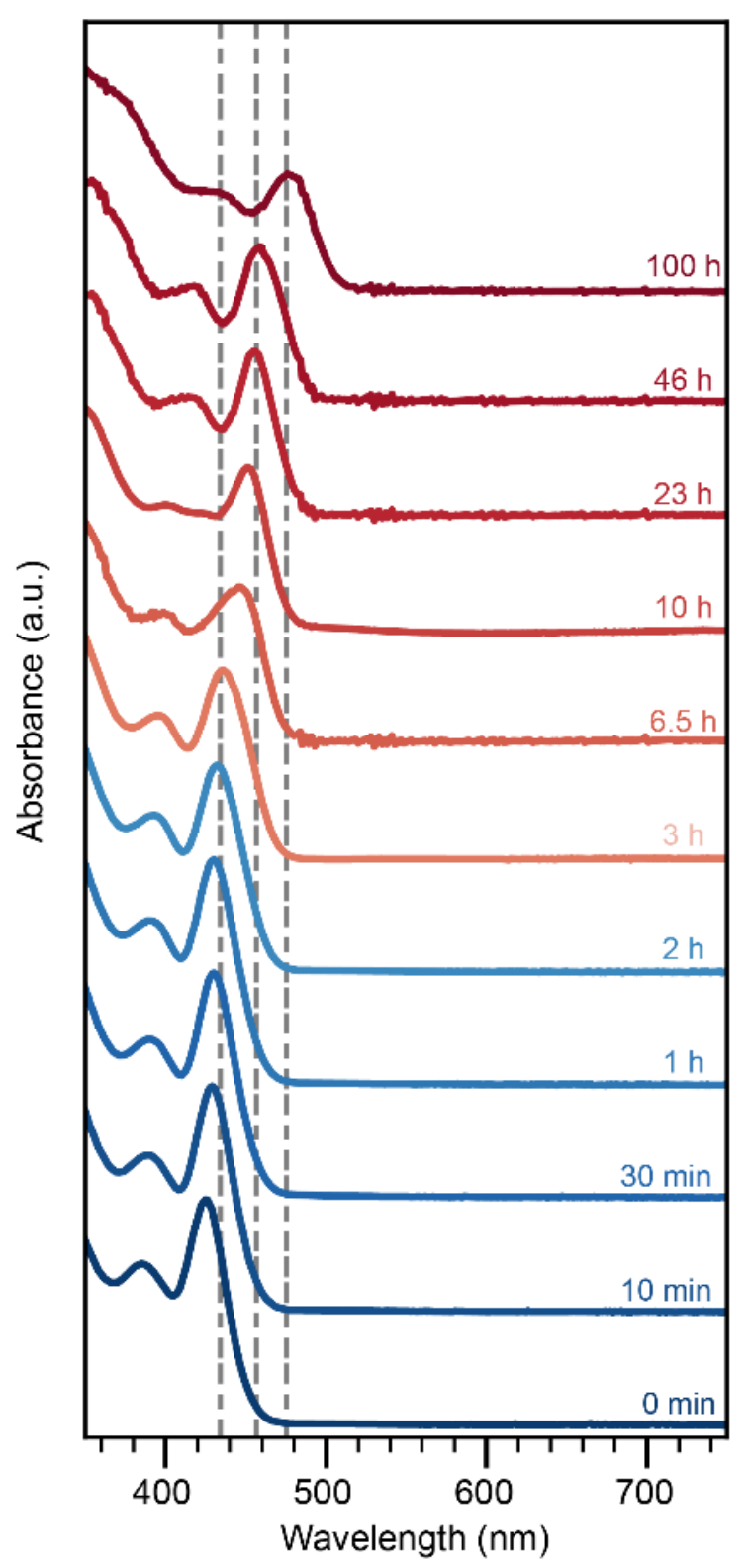

Figure S10. Growth of isolated CdSe MSNC 434 without selenium and cadmium precursors. Temporal evolution of the optical absorption spectra of the isolated 434 heated in ODE at $180^{\circ} \mathrm{C}$. Each spectrum is labeled with the growth time. The vertical dashed lines indicate the lowest energy excitonic peaks for three MSNCs (at 434, 455, and $476 \mathrm{~nm}$ ). Discrete growth is not observed. 
a

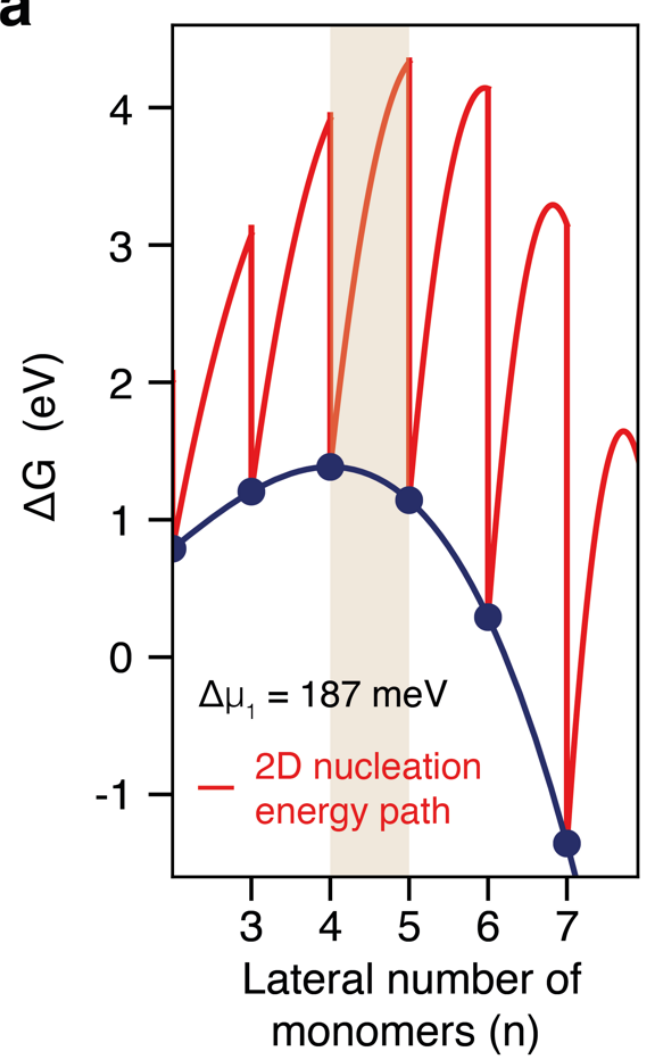

b

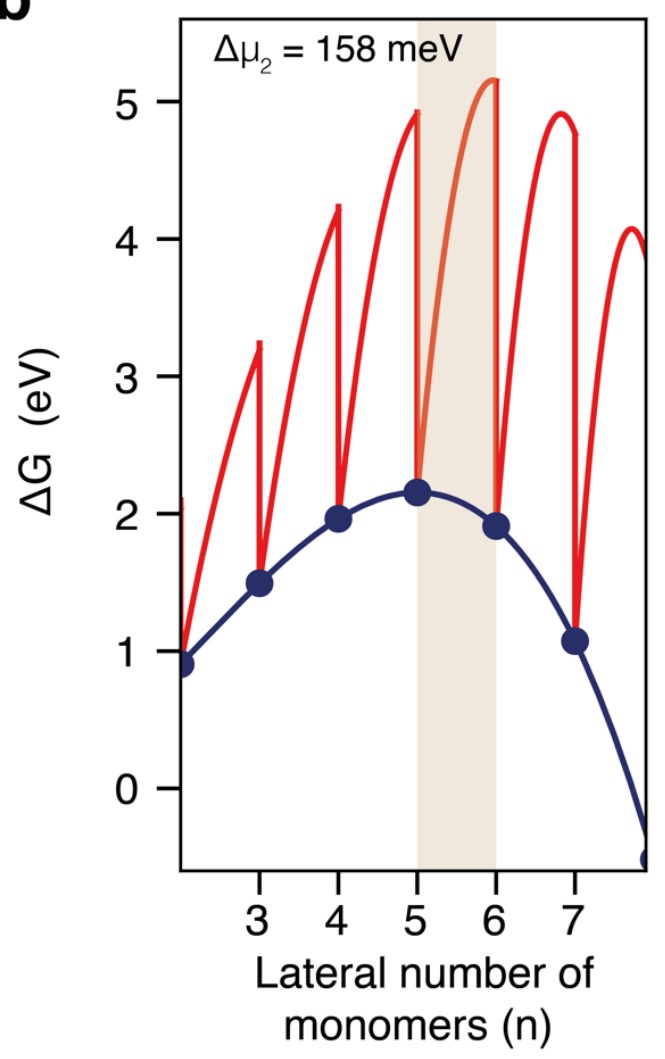

Figure S11. Free energy of formation for different-sized MSNCs for two different fixed supersaturations $(\Delta \mu)$. $(\mathrm{a}, \mathrm{b})$ The total free energy of different sizes of MSNCs (blue dots) and the transition states (red lines) that link them are plotted for different supersaturations $[\Delta \mu$ in (a) is larger than in (b)]. As the supersaturation decreases, the barriers linking different MSNC sizes increase. Simultaneously, the size range of stable MSNCs exhibiting size-dependent barriers (highlighted in beige) shifts to larger MSNC sizes. 
a

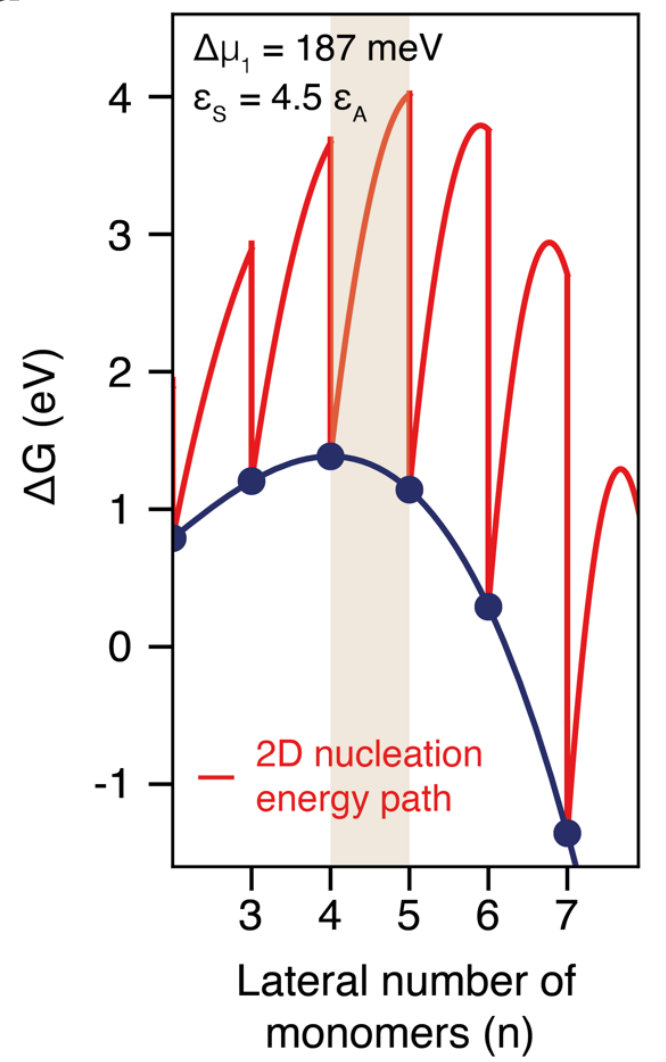

b

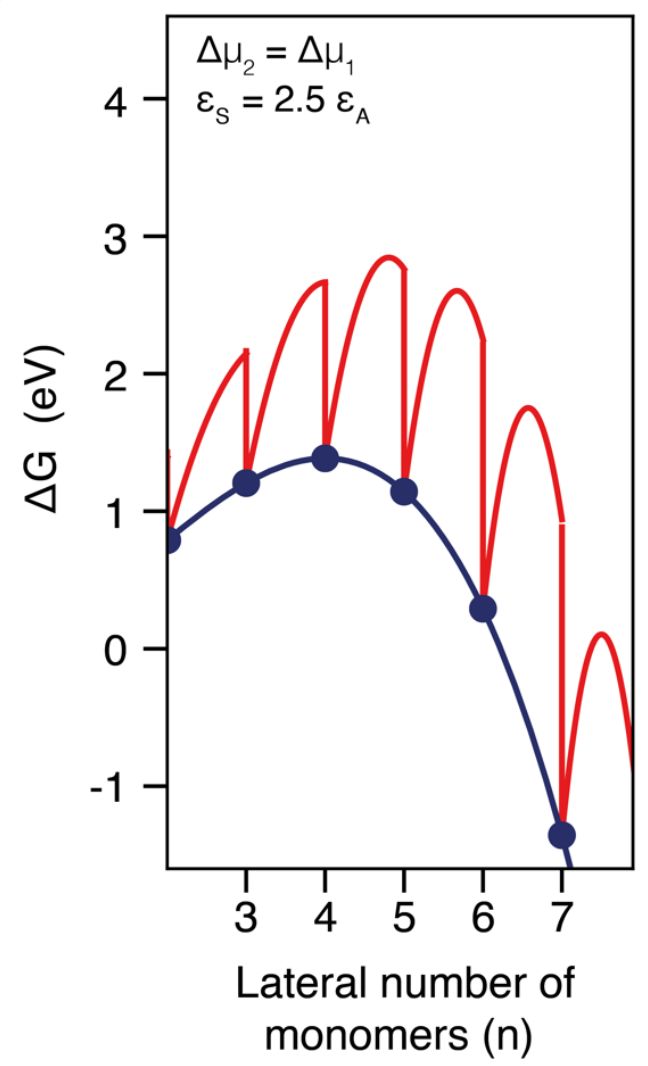

Figure S12. Free energy of formation for different-sized MSNCs for different model parameters. $(a, b)$ Total free energy of different sizes of MSNCs (blue dots) and the transition states (red lines) that link them are plotted for (a) $\varepsilon_{S}=4.5 \varepsilon_{A}$ and (b) $\varepsilon_{S}=2.5 \varepsilon_{A}$. The supersaturation and surface energy, $\varepsilon_{A}$, is the same in both plots. Changes in $\varepsilon_{S}$ do not affect the absolute stability of MSNCs (eq S7), they only affect the barriers linking different sizes (eq S9). When $\varepsilon_{S}$ does not fulfill the inequality given in eq S13 [as in (b) where $\varepsilon_{S}=2.5 \varepsilon_{A}$ ], no size-regime exists for stable MSNCs exhibiting size-dependent barriers. However, MSNCs still correspond to local minima in the energy of formation curve. 

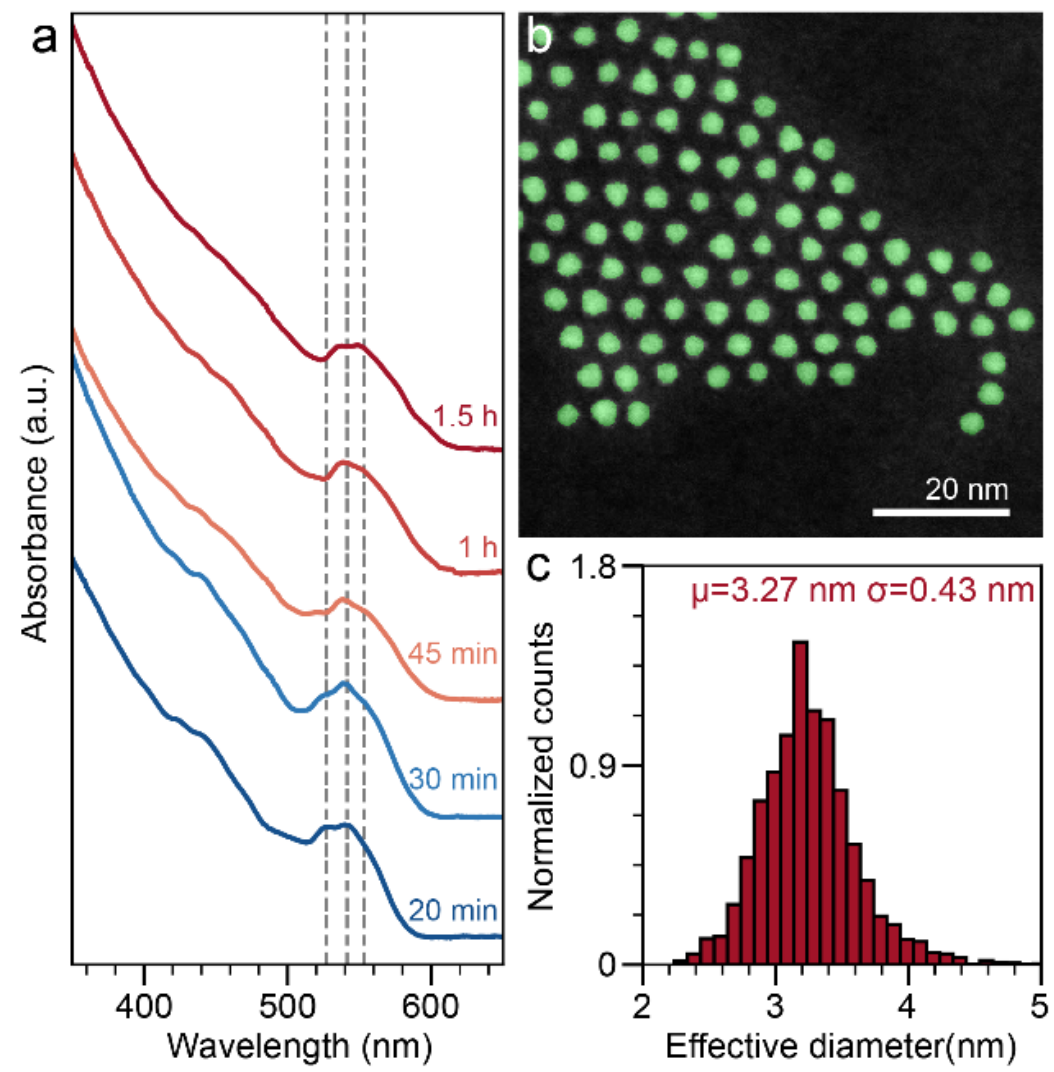

Figure S13. Optical characterization and size analysis of CdSe MSNCs grown in the presence of chlorides. (a) Temporal evolution of the optical absorption spectra of CdSe MSNCs grown at $240{ }^{\circ} \mathrm{C}$ in the presence of chloride. Each spectrum is labeled with the time after injection. The vertical dashed lines indicate the lowest energy excitonic peaks for three MSNC product species (at 528, 542, and $554 \mathrm{~nm}$ ). At least two new MSNC species were observed. We note that a slight red shift occurs for the spectral position of the absorption peaks of the larger MSNC sizes when chloride is present (528 versus $523 \mathrm{~nm}$ ). See also Figure 6 in the main text. (b) Image shows detected objects (green) after evaluating the high-angle annular dark-field (HAADF) scanning transmission electron microscopy (STEM) images of 554 species using a custom Python script. While efforts were taken to reduce beam damage in these images (see section S1.3), the shape of the particles may have evolved during the exposure. (c) Normalized histogram with the mean $(\mu)$ and standard deviation $(\sigma)$ of the extracted effective diameter of the 554 species. A total of 4,517 particles were analyzed. The bin size of the histogram is $0.1 \mathrm{~nm}$. 


\section{S6. SUPPORTING REFERENCES}

(S1) Hendricks, M. P.; Campos, M. P.; Cleveland, G. T.; Jen-La Plante, I.; Owen, J. S. A Tunable Library of Substituted Thiourea Precursors to Metal Sulfide Nanocrystals. Science 2015, 348 , $1226-1230$.

(S2) Koketsu, M.; Nada, F.; Hiramatsu, S.; Ishihara, H. Reactions of Acyl Chlorides with LiAlHSeH. Preparation of Diacyl Selenides, Diacyl Diselenides, Selenocarboxylates and Cyclic Selenoanhydrides. J. Chem. Soc., Perkin Trans. 1 2002, No. 6, 737-740.

(S3) Beecher, A. N.; Yang, X.; Palmer, J. H.; Lagrassa, A. L.; Juhas, P.; Billinge, S. J. L.; Owen, J. S. Atomic Structures and Gram Scale Synthesis of Three Tetrahedral Quantum Dots. J. Am. Chem. Soc. 2014, 136, 10645-10653.

(S4) Kudera, S. Formation of Colloidal Semiconductor Nanocrystals: The Aspect of Nucleation, Ph.D. Thesis, Ludwig-Maximilians-Universität München, October 2007.

(S5) Ko, J. H.; Yoo, D.; Kim, Y. H. Atomic Models for Anionic Ligand Passivation of CationRich Surfaces of IV-VI, II-VI, and III-V Colloidal Quantum Dots. Chem. Commun. 2017, 53, 388-391.

(S6) Liu, L.; Zhuang, Z.; Xie, T.; Wang, Y.-G.; Li, J.; Peng, Q.; Li, Y. Shape Control of CdSe Nanocrystals with Zinc Blende Structure. J. Am. Chem. Soc. 2009, 131, 16423-16429.

(S7) Bealing, C. R.; Baumgardner, W. J.; Choi, J. J.; Hanrath, T.; Hennig, R. G. Predicting Nanocrystal Shape through Consideration of Surface-Ligand Interactions. ACS Nano 2012, 6 , $2118-2127$.

(S8) Riedinger, A.; Ott, F. D.; Mule, A.; Mazzotti, S.; Knüsel, P. N.; Kress, S. J. P.; Prins, F.; Erwin, S. C.; Norris, D. J. An Intrinsic Growth Instability in Isotropic Materials Leads to Quasi-Two-Dimensional Nanoplatelets. Nat. Mater. 2017, 16, 743-748. 